Melanie Tatur

\title{
Ökonomische Transformation, Staat und moralische Ressourcen in den post-sozialistischen Gesellschaften
}

Die Einsicht, daß politisch definierte Institutionen und deren kulturelle Einbettung für ökonomische Produktivität und Wirtschaftswachstum von wesentlicher Bedeutung sind, gilt heute als eine Binsenweisheit, und der Verweis auf »politische Rahmenbedingungen «, »frames « der Interessenwahrnehmung und »Werte « fehlt nicht mehr in den Analysen zur ökonomischen Transformation (Höhmann 1995, Bönker 1995, Kornai 1996). Dennoch bleiben auch die sozialwissenschaftlichen Betrachtungen ökonomischer Transformation insofern den ökonomistischen Denktraditionen der Politischen Ökonomie und ihrer Annahme eines »rational choice« verbunden, als Transformation selber nicht institutionalistisch - als politischkulturelle Innovation des Reproduktionsmodus der sozialistischen bzw. post-sozialistischen Gesellschaft - konzeptualisiert wird. Das gilt - wie ich argumentieren werde - vor allem auch für Verfechter einer »evolutionistisch-gradualistischen « Perspektive und Politik, d.h. für Wissenschaftler und Politiker, die sich als sozialdemokratische oder sozialistische »Institutionalisten« verstehen.

Ich möchte in diesem Beitrag das von Jadwiga Staniszkis entwickelte Konzept des »politischen Kapitalismus « - als einer hybriden gesellschaftlichen Formation und eines institutionellen Modus der Umgestaltung der sozialistischen Gesellschaft unter Bedingungen einer peripheren Lage (Staniszkis 1995) - zu einem Kategorienapparat ausbauen, der vergleichende Analysen von Transformationspfaden in Mittel- und Osteuropa anleiten kann. Dabei versuche ich unterschiedliche Formen des »politischen Kapitalismus« über den Charakter des Staates zu definieren und mit kulturellen Integrationsmustern der Gesellschaften in Verbindung zu bringen. Der Beitrag versteht sich so auch als ein erster Versuch, den von Staniszkis entwickelten Begriff um eine kulturelle Dimension zu erweitern, die in meiner Konzeptualisierung den entscheidenen Faktor der Diversifizierung von Transformationspfaden bildet ${ }^{1}$.

1 Auch Staniszkis verweist auf kulturelle Unterschiede, die unterschiedliche Transformationspfade mitbestimmen. Die Abstraktions- und Vergleichsebene ist aber eine andere, nämlich die der unterschiedlichen Pfade von Mitteleuropa, Rußland und China und der 
Ich entwickle meine Kategorien in folgenden Schritten. Als erstes (1.) versuche ich »Transformation « und »politischen Kapitalismus « unter Rückgriff auf die wirtschaftshistorische Theorie der »Eigentumsrechte« (Thomas/North 1973, North 1990) politisch - als Verhältnis von Staat und Gesellschaft - zu bestimmen. Dann (2.) erweitere ich das Konzept, indem ich einen Perspektivwechsel vollziehe und globalisierungspolitische Diskurse um die politischen Bedingungen ökonomischer Wettbewerbsfähigkeit (Porter 1990, Messner/Meyer-Stamer 1993) sowie Überlegungen über kulturelle Voraussetzungen der Effizienz demokratischer Institutionen (Putnam 1993) in die Überlegungen einbeziehe. Dabei hebe ich die kulturelle Dimension des "politischen Kapitalismus « hervor, indem ich die begriffliche Unterscheidung von »amoralischem Familiarismus « (Banfield 1958) und »ziviler Gemeinschaft« (Putnam 1993) einführe und durch ein Konzept tribalistisch fragmentierter Moral ergänze. Nachdem ich die Überlegungen in einer idealtypischen Begriffskonstruktion von »kriminellem«, »politischem « und »demokratischem « Kapitalismus zusammengefaßt habe, versuche ich (3.), das zunächst statische Schema über die Illustration unterschiedlicher Transformationspfade und deren sozialstruktureller Konsequenzen in verschiedenen Ländern Osteuropas zu dynamisieren und so »politische Kapitalismen« als Modi der Transformation zu fassen.

\section{Transformation und Transformationsstrategien - \\ »Sozialismus«, »demokratischer Kapitalismus«, »politischer Kapitalismus « - »Gradualismus« und »Schocktherapie«}

In der sozialwissenschaftlichen Diskussion um die Transformationen in Ost- und Mitteleuropa lassen sich bis in die Mitte der neunziger Jahre zwei Perspektiven unterscheiden. Die neuen liberalen, demokratischen Eliten formulierten 1990 das Projekt einer »offenen Gesellschaft«. Eine neue demokratische und marktwirtschaftliche Ordnung sollte »von oben « über Rechtsetzung und Politikstrategien implementiert werden. Auch in der sozialwissenschaftlichen Diskussion fand diese Perspektive einen Niederschlag: So formulierte Ralf Dahrendorf schon 1990 seinen Phasenplan von Verfassungsgebung, politischer Ausgestaltung von politischem System und Wirtschaftsordnung und kultureller Anpassung und setzte für die einzelnen Etappen sogar zeitliche Limits - Monate, Jahre und Jahrzehnte - an. Skeptischere Stimmen verwiesen auf das zivilisatorische Erbe des Sozialismus, das solch eine Anpassung unwahrscheinlich oder doch problematisch mache (Sztompka 1993, Srubar 1994) und auf immanente Dilemmata des Projektes der demokratischen Transformation sozialistischer Gesellschaften.

jeweiligen Kultursysteme. Mir geht es darum, ein begriffliches Instrumentarium zu entwickeln, das es erlaubt, die Entwicklungen im post-sozialistischen Europa in Hinblick auf den Typus des post-sozialistischen Kapitalismus zu diversifizieren und zu vergleichen. 
So sprach Staniszkis schon in den achtziger Jahren von der »Akteurslücke« (1991a), und Offe (1994) machte auf das Dilemma »gleichzeitigen« Nachholens von Prozessen mit unterschiedlicher Zeittiefe (Nationenbildung, Verfassungssetzung und Alltagspolitik) aufmerksam. Gemeinsam war den Reformkonzepten der neuen Eliten und den Überlegungen der Sozialwissenschaftler der Bezug auf das Projekt der Transformation, d.h. ein Modell des entwickelten demokratischen Kapitalismus, aus dem Begriffe für die strategischen Entwürfe und Analysen der Dilemmata abgeleitet wurden.

Schon früh haben Soziologen wie David Stark (1991, 1994) und Jadwiga Staniszkis (1991) gegen die implizite Annahme vom »Zusammenbruch« der alten Ordnung polemisiert, den Glauben an eine politisch-legalistische Methode institutionellen Wandels als illusionär kritisiert und auf Kontinuitäten im Wandel verwiesen. Aufgelöst hätten sich - so Stark unter Verweis auf Ungarn - bestenfalls die politischen Fassaden der alten Ordnung, dahinter aber befände sich keineswegs ein gesellschaftliches Vakum, sondern das soziale Gewebe von informellen Beziehungen, Regeln, Routinen, das die sozialistische Gesellschaft getragen habe. »Transformation« sei nicht als Projekt, sondern als graduelle Evolution der sozialistischen Ordnung zu begreifen. Staniszkis konstatiert demgegenüber - am polnischen Beispiel einen widersprüchlicheren Prozeß der Kontinuität im Wandel und des Wandels in der Kontinuität: Wie Stark verwies sie auf Kontinuitäten in der Dimension informeller Verhaltensstandards und Beziehungsnetze und darauf, daß diese die institutionellen Innovationen »von oben « unterlaufen; zugleich aber zeigte sie, daß die Einführung neuer demokratischer Spielregeln und neuer makroökonomischer Rationalitätsstandards (Schocktherapie und Öffnung) die Bedeutung und Funktion der überkommenen Muster und Akteurskonstellationen verändert und vor allem eine neue Entwicklungsdynamik eingeleitet habe.

Bei diesen scheinbar geringfügigen Differenzen handelte es sich nur oberflächlich um länderspezifische Besonderheiten. Zwar stimmen Stark und Staniszkis insoweit überein, als sie die Transformation als Evolution der sozialistischen Gesellschaft betrachten, in der Bewertung der konstatierten Kontinuität im Wandel unterscheiden sie sich aber deutlich. Während Stark von der Herausbildung einer »Mischwirtschaft« spricht, deren Merkmale »hybride Eigentumsformen« und »kooperative Netzwerke« seien und entsprechenden Phänomenen in hochentwickelten kapitalistischen Ökonomien ähnelten (Stark 1996), konstatiert Staniszkis die Konstitution eines »politischen Kapitalismus « als einer spezifischen post-sozialistischen Formation, deren »hybride Eigentumsformen « und informelle Vernetzungen der ökonomischen und politischen Verwaltungen nur äußerlich, - bzw. nur in begrenztem Umfang - entsprechenden Phänomenen in modernen westlichen Gesellschaften ähnelten und denen tatsächlich die Funktion zukomme, 
staatliche Ressourcen zu transferieren, Kosten privater Gewinne über den Staat zu externalisieren oder exklusive Zugänge zu einer staatlich gestützten finanziellen Infrastruktur zu monopolisieren. »Politischer Kapitalismus« ist bei Staniszkis ein Modus der Transformation, genauer ein institutionelles Muster, über das privatwirtschaftliche Akteure und Kapital geschaffen werden. Dieses Urteil deckt sich mit Aslunds Analyse. Der liberale schwedische Wirtschaftswissenschaftler konstatiert für Rußland unterschiedliche Formen des »rent-seeking «, d.h. die Kontinuität des Mechanismus zentraler Umverteilung, dem aber unter neuen Bedingungen die Funktion privater Gewinnaneignung und Kapitalkonzentration zukommt (Aslund 1995, 1996). Während Aslund die Mechanismen des »rent-seeking « als Übergangsphänomene klassifiziert und auf eine letztliche Durchsetzung von Orientierungen des »profit-seeking « in kompetitiven Märkten hofft, haben andere Autoren für Rußland die Transformation zu vermachteten QuasiMärkten hervorgehoben (s.u.). Auch Staniszkis betrachtet für Polen und Mitteleuropa den »politischen Kapitalismus« als eine Übergangsformation. Als institutioneller Modus der wirtschafts- und sozialstrukturellen Anpassung hinterläßt er aber dauerhafte Spuren in der zukünftigen sozialen Struktur in Gestalt eines mehr oder weniger peripheren Kapitalismus. Dabei verweist sie auf eine historische Analogie. Ähnlich wie sich der grundbesitzende polnische Adel, der im 16. Jahrhundert mit dem westeuropäischen Getreidemarkt in Berührung kam, als soziale Gruppe in der neuen Situation dadurch behaupten konnte, daß er - unter Bedingungen des Warenverkehrs - auf die feudale Institution der Leibeigenschaft zurückgriff und damit einen Entwicklungspfad programmierte, der die Peripherisierung des Landes bedeutete, ermögliche es der Rückgriff auf institutionelle Muster des Sozialismus zwar lokalen Akteuren, sich unter den neuen Bedingungen der Integration des Landes in die Weltwirtschaft in der neuen Umwelt zu behaupten; die institutionellen Weichenstellungen bedeuteten aber für die Gesellschaften eine Blockade ihrer Entwicklungspotentiale (Staniszkis 1995).

Den unterschiedlichen Bewertungen der Kontinuität im Wandel der postsozialistischen Gesellschaften liegen - nach meiner Einschätzung - tiefer gehende Divergenzen im begrifflichen Verständnis der reifen sozialistischen Ordnung zu Grunde. Mit ungarischen Sozial- und Wirtschaftswissenschaftlern, deren Denken stark von der technokratischen Reformpolitik seit den sechziger Jahren geprägt ist, faßt Stark den Sozialismus als ein System zentraler Redistribution und die Reformen seit Beginn der sechziger Jahre als einen stetigen Prozeß des Umbaus dieser Form von Wirtschaftsorganisation, durch die Hereinnahme von Marktmechanismen und eine Kombination von »bürokratischer « und »marktförmiger« »Koordination« (Kornai 1995). Diese Modifikationen des Leitungssystems haben 
nach Stark zur Folge, daß den Managern der staatlichen Betriebe faktisch »Eigentumsrechte« zuwüchsen (Stark 1991), und im Rahmen der alten Ordnung - mit Marktkoordination und Verfügungsmacht der Manager bereits eine neue Ordnung heranwachse.

Demgegenüber faßt Staniszkis den Sozialismus nicht als einen Typus von Wirtschaftsorganisation, sondern als eine politisch-staatliche Ordnung. Konstitutives Merkmal ist nicht der ökonomische Mechanismus der zentralen Redistribution, sondern die politische Atomisierung der Gesellschaft, ihre Ersetzung durch administrative Strukturen. Das hat nicht nur Funktionsdefizite im ökonomischen Bereich zur Folge, sondern definiert eine andere Reproduktionslogik der Gesellschaft als einer Formation. Die Logik des sozialistischen Systems ist eine Logik der Reproduktion von Macht und Abhängigkeit - nicht wie die der kapitalistischen Gesellschaft eine der Reproduktion von Reichtum und Ausbeutung (Staniszkis 1992). In ähnlicher Weise hat der amerikanische Soziologe A.C. Janos die sowjetische Ordnung als »Militärstaat« im Sinne Herbert Spencers charakterisiert (Janos 1991) - als eine Formation, so könnte man sagen, in die die Reproduktionslogik des state-building (Tilly) und nicht die des Kapitals eingegeben ist. Das heißt keineswegs, daß das sozialistische System einfach mit dem totalitären Gewaltapparat identisch wäre, über den es implementiert wurde, sondern daß auch im reifen, post-stalinistischen Sozialismus - als einem sich selbst tragenden Geflecht von Beziehungen, das die atomisierten, durch den Staat vergesellschaften Menschen in Form von Alltagsroutinen, Denkweisen und Interessen reproduzierten - das Primat der politischen Macht und der politischen Stabilität institutionalisiert war. ${ }^{2}$ Die Reformen des Post-Stalinismus erscheinen aus dieser Perspektive als »Deartikulationen « des Systems (Staniszkis 1991) d.h. als Hereinnahme fremder Elemente ${ }^{3}$, die aber deshalb nicht den Beginn einer »Mischwirtschaft« bedeuten, weil sie der Herrschaftslogik des Systems untergeordnet blieben.

2 Entsprechend könnte man Kornai (1996) erwidern, daß auch die von ihm konstatierten Merkmale des kadaristischen »Gulaschkommunismus«, die er als »Konsumorientierung « und sozialstaatlichen »Staatspaternalismus« bezeichnet und als Reflex der Machteliten auf das Trauma der Revolution von 1956 sowie eingeübte Wertorientierungen bzw. Erwartungshaltungen der Bevölkerung erklärt, Ausdruck der dominanten (paternalistisch gefaßten) Herrschaftsrationalität waren. Das Problem des »Gulaschkommunismus « waren aber nicht - wie Kornai glauben macht - die Erwartungshaltungen der Bevölkerung, sondern der Umstand, daß ein in erster Linie an der eigenen Reproduktion interessierter politökonomischer Apparat diese nicht (oder nur über Verschuldung) befriedigen konnte.

3 Als Deartikulation können die Öffnung Polens und Ungarn zum Weltmarkt bei Beibehaltung des staatlichen Außenhandelsmonopols und der Staatsbanken, die Legalisierung des privaten Sektors in Polen seit der Mitte der fünfziger Jahre und in Ungarn in den sechziger Jahren (wobei diese aber in den staatlichen Apparat eingebettet und durch Lizenzvergabe und Aufsicht, sowie den Nischencharakter im Verhältnis zur staatlichen Ökonomie von dieser abhängig blieben) betrachtet werden. Hierzu gehört auch die Herausbildung einer Opposition, deren Status aber informell, d.h. rechtlich ungesichert blieb (Staniszkis 1992). 
Der unterschiedlichen Bewertung der Kontinuität im Wandel liegen schließlich auch divergierende implizite Annahmen über die konstitutierenden Bedingungen des westlichen demokratischen Kapitalismus zugrunde. Zwar berufen sich Stark und Staniszkis gleichermaßen auf die Property RightsSchule, deuten deren Annahmen und Forschungsergebnisse aber unterschiedlich.

Wenn Stark auf faktische »Eigentumsrechte« verweist, die dem ungarischen Management mit den Reformen seit den sechziger Jahren zugewachsen seien, wenn er affirmativ von einer »Rekombination« von Eigentum (Stark 1994) spricht und die neuen hybriden Eigentumsformen und informellen Netzwerke mit denen eines modernen kooperativen Kapitalismus gleichsetzt, betrachtet er auch hier die Wirtschaftsordnung als einen Koordinationsmechanismus und Eigentumsrechte als Verfügungsmacht unterschiedlicher Akteure. Für die Frage der »Eigentumsrechte« erscheint der Umstand nicht relevant, daß auch das ungarische Management bis 1989 der Nomenklatur unterstand und das politische System - wie andernorts auch die Prärogative der Partei sicherte. Tatsächlich bedeutete dies, daß die Verfügungsmacht im reformierten ungarischen Sozialismus zwar in stärkerem Maße beim wirtschaftlichen Verwaltungsapparat gebündelt war als in anderen Ländern, nicht aber, daß »Eigentumsrechte« die institutionelle Grundlage der ökonomischen Beziehungen bildeten.

Für die Entstehung der »westlichen Welt « und der sie charakterisierenden Wirtschaftsdynamik ist aber die Institutionalisierung von Eigentumsrechten von entscheidender Bedeutung gewesen. Eigentumsrechte meinten in diesem Zusammenhang keineswegs nur Verfügungsmacht, sondern Rechte, d.h. die rechtliche Verankerung ökonomischer Macht und die politische Konstitution ökonomischer Anreizsysteme durch den sich aus einer nun privaten ökonomischen Sphäre zurückziehenden Staat. Das Neue bei der historischen Erfindung der »Eigentumsrechte« war nicht die Verfügungsmacht des englischen Adels, sondern deren rechtliche Verankerung und staatliche Sanktionierung. Das heißt, die institutionelle Innovation war die Rekonstitution des Staates als einer öffentlichen Ordnung, die einen gesellschaftlichen Raum privater Wirtschaftstätigkeit freigab und zugleich institutionalisierte (Thomas/North 1973, North 1990). Die Geltung von dauerhaften, ausschließlichen und veräußerbaren Eigentumsrechten sicherte die eindeutige Zuordnung der Kosten und Nutzen des Handelns und schuf so eine Anreizstruktur für Markterweiterung und endogenes Wirtschaftswachstum. Die Stärke bzw. Schwäche von Eigentumsrechten, die ökonomisch erfolgreiche bzw. erfolglose Entwicklungspfade begründete, ist somit nicht eine Frage des Eigentums und der Verfügungsmacht, sondern der politischen und gesellschaftlichen Ordnung. Am Beispiel Englands und Spaniens, den USA und Lateinamerikas verdeutlicht North die Konstitution 
von zwei divergierenden politisch-ökonomischen Ordnungen und darüber institutionalisierten »Entwicklungspfaden «: Die mit der Veränderung der Militärtechnologie im 16. Jahrhundert einhergehende Krise des Staatshaushaltes wurde in England durch einen Ausgleich zwischen Ständen und Krone und eine darüber geschaffene institutionelle Innovation gelöst: die durch Parlament und Justiz gesicherte Herrschaft des Gesetzes garantierte Eigentumsrechte und darüber ein Anreizsystem, das die Erweiterung der Märkte, Orientierungen des profit-seeking und endogenes Wirtschaftswachstum begünstigten. In Spanien dagegen setzte sich ein Politikmuster durch, das über Dekrete und Eingriffe die wirtschaftliche Tätigkeit den Interessen des Fiskus untergeordnet beließ, was zur Erweiterung staatlicher Apparate, zu ökonomischen Orientierungen am rent-seeking und schließlich zu wirtschaftlichem Niedergang führte. North kontrastiert dabei nicht nur zwei politische Ordnungstypen, er zeigt auch die Reproduktionsfähigkeit von Institutionen und eine darüber begründete Pfadabhängigkeit ökonomischen Wandels. Denn die zunächst historisch zufälligen institutionellen Optionen definierten Orientierungen und Kräfteverhältnisse und objektivierten sich als Systeme und soziale Strukturen. Aus dieser Perspektive meint ökonomische Transformation nicht die Einführung von »Marktkoordination « und privatem Eigentum, sondern die Institutionalisierung von Eigentumsrechten als institutioneller Grenzsetzung zwischen öffentlicher und privater Handlungssphäre - über Recht und eine demokratische Ordnung.

Die unterschiedlichen paradigmatischen Vorentscheidungen über »Sozialismus «, »Transformation« und »demokratischen Kapitalismus« schlagen sich auch in den Kontroversen um konkurrierende Transformationsstrategien nieder. In der Diskussion zwischen »Gradualisten« und Anhängern der »Schocktherapie« ging es weniger um eine Kontroverse zwischen »Institutionalisten « und »neo-liberalen « Monetaristen als um die Konfrontation zweier Paradigmata der Transformation. Von den Vertretern des »Gradualismus « wurde die Kontroverse um die angemessenere Transformationspolitik als eine Frage der zeitlichen Streckung und Abfederung der sozialen Kosten der Herstellung des ökonomischen Gleichgewichts, der strukturpolitischen Anpassung und des Tempos der Privatisierung behandelt. Dabei wurde - als Ausdruck einer »politischen Ökonomie« - dem Verhältnis von politischer Entscheidungsfähigkeit der Regierung und potentiellem Widerstand der Bevölkerung eine zentrale Bedeutung beigemessen (Kornai 1996, Rosati 1993) und die gewählten politischen Reformstrategien mit dem Umfang gesellschaftlicher Unterstützung für Regierung und Reform bzw. der Konfliktfähigkeit von Entscheidungsträgern und Bevölkerung in Verbindung gebracht (Bönker 1995; Kornai 1996).

Die »neoliberalen« Befürworter der »Schocktherapie« argumentierten dagegen nicht in Kategorien einer Ökonomie der Zeit, sondern des institutio- 
nellen Wandels. Mit Hilfe eines Bündels von makroökonomischen und politischen Maßnahmen sollte eine neue Anreizstruktur für das Wirtschaftshandeln geschaffen werden. Makroökonomische Stabilisierung, Liberalisierung und institutionelle Innovation bildeten ein Paket von Instrumenten zur Revolutionierung der Rationalitätsstandards des Handelns. Der politische Mechanismus vertikaler »bürokratischer Koordination « sollte außer Kraft gesetzt und durch horizontale Marktbeziehungen ersetzt werden. Hiermit sollten die Voraussetzungen für einen nicht nur rechtlich, sondern auch hinsichtlich der ökonomischen Chancen »freien« Markteintritt für neue Wirtschaftssubjekte geschaffen und das Handeln aller Akteure von Orientierungen des rent-seeking gegenüber dem umverteilenden Staat auf profit-seeking am Markt umorientiert werden. ${ }^{4}$ Der »Schock« war somit nicht an die Bevölkerung, sondern an das staatliche Management adressiert, für das die Rationalitätsstandards des Handelns (und der Nutzung ihrer Verfügungsrechte) abrupt umgestellt wurden. Das heißt natürlich nicht, daß die Umstellung der Rationalitätsstandards keine sozialen Kosten verursacht hätten. Das Argument der »Neoliberalen« war aber, daß eine radikale Umformulierung der institutionellen Handlungsbedingungen die Anpassungsrationalität des Gesamtsystems gegenüber der Weltwirtschaft erhöhen würde. Aus dieser Perspektive wurde der »Gradualismus« nicht deswegen kritisiert, weil er Öffnung und Strukturanpassung zu langsam vollzog, sondern weil er - ungeachtet der formalen rechtlichen Neuerungen - den ökonomischen Funktionsmechanismus nicht hinreichend schnell umformuliere, damit die Standards wirtschaftsstruktureller Anpassung nicht revolutioniere

4 Der Balcerowicz-Plan war ein Maßnahmepaket, das auf makroökonomische Stabilisierung, Liberalisierung und institutionellen Wandel abzielte, wobei makroökonomische Maßnahmen, rechtliche und institutionelle Veränderungen ein komplementäres Gefüge bildeten. Das Programm bezog sich auf die Spezifik der polnischen Situation: eine anhaltende Strukturkrise, eine makroökonomische Katastrophe (Hyperinflation) und die politische Ressource einer Gemeinwohlverpflichung der Bevölkerung als Erbe der Solidarnsoc (Balcerowicz 1995, 290ff). Nach vorbereitenden Maßnahmen 1989 wurde das Programm 1990 eingeleitet. Die wichtigsten Maßnahmen zur Stabilisierung waren die Reduktion des Haushaltsdefizits, die Kontrolle der Geldversorgung, die Lohnkontrolle und die Festsetzung eines fixen Wechselkurses. Die Liberalisierung beinhaltete die Beseitigung der massiven und detaillierten Staatsinterventionen, die Aufhebung zentraler Allokationen, die nahezu vollständige Freigabe der Preisbildung, die Beseitigung der verbliebenen Restriktionen für private Wirtschaftstätigkeit, die Liquidierung der Import- und Exportrestriktionen sowie die Durchsetzung der inneren Konvertibilität der Währung. Die institutionelle Restrukturierung beinhaltete eine Antimonopolgesetzgebung, die institutionelle Unabhängigkeit der Zentralbank, die Reform von Bank und Versicherungswesen, die Steuerreform und die Schaffung einer genuinen lokalen Selbstverwaltung. Die Strukturanpassung der polnischen Wirtschaft sollte sich spontan, über den Druck der neuen makroökonomischen Rahmenbedingungen vollziehen. Hierdurch schien den Reformern der angestrebte institutionelle Bruch mit dem System der zentralen Umverteilung und seine Reproduktion in einer Rentenökonomie möglich. Allein für den Bergbau, die Energiewirtschaft und die Eisenverhüttung wurde eine staatliche Strukturpolitik angestrebt (Balcerowicz 1995, 318f). 
und deshalb - wegen des andauernden Primats politischer Macht gegenüber ökonomischer Rationalität - die gesellschaftlichen Kosten dieser Anpassung auf lange Sicht erhöhe. ${ }^{5}$ Mit anderen Worten: Die neoliberalen »Monetaristen« und nicht die sich als »Institutionalisten« ausweisenden Sozialdemokraten waren die besseren Institutionalisten.

Das ist insofern nicht verwunderlich, als hinter der Kontroverse zwischen Gradualisten und Neoliberalen identifizierbare korporative bzw. Gruppeninteressen und Denktraditionen standen. Die »neoliberale« Position eines komplexen institutionellen Bruchs wurde von den neuen demokratischen Gegeneliten, die »gradualistische« von der postkommunistischen Sozialdemokratrie vertreten. Das gilt mit Abstrichen auch in Ungarn, wo es auf den ersten Blick die »neuen« Eliten der konservativen Regierung waren, die den »Gradualismus « favorisiert hatten, und die 1994 gewählte von den postkommunistischen Sozialisten dominierte Koalition seit 1995 einer »Schocktherapie« das Wort redete. Die gradualistische Reformpolitik wurde in Ungarn nicht nur schon unter dem Reformkommunismus länger vorbereitet und eingeleitet, der Gradualismus wurde auch von einer »neuen« Regierungspartei betrieben, die kein eigenes Wirtschaftsprogramm und einen postkommunistischen Finanzminister hatte. Die »Schocktherapie« der Sozialisten bezog sich nach deren Selbstverständnis allein auf konjunkturpolitische und sozialpolitische Maßnahmen zur Einschränkung des Massenkonsums und der staatlichen Transfersleistungen an die Bevölkerung. Das technokratische Reformdenken war und ist in Ungarn politisch nicht kontrovers. ${ }^{6}$ Schon die »neue konservative « Regierung versuchte sich nach dem Fehlschlag symbolischer Legitimierung wie ihre Vorgängerin und ihre Nachfolgerin über Patronage und Klientelpolitik im Wirtschaftsapparat zu verankern.

5 Wie richtig dieses Argument ist, zeigen die Ergebnisse der »gradualistischen « Reformpolitiken in Bulgarien und Rumänien und selbst die erstaunlichen Probleme der ungarischen Wirtschaft.

6 Es ist so auch nur auf den ersten Blick frappierend, daß in Ungarn, wo sich nicht nur vor 1989 eine Vielzahl von Techniken des rent-seeking etabliert hatten, sondern diese auch ungebrochen in die neue Zeit übernommen und institutionell ausgebaut wurden, ein kritischer Begriff des »politischen Kapitalismus« als eines institutionellen Phänomens nicht entwickelt wurde, während dies in Polen geschah, wo die liberale Politik 1989-1993 die »spontane Privatisierung « blockierte und die Techniken des rent-seeking in den Bereich der Illegalität und Halblegalität von »Affairen« verbannte (s.u.). In Ungarns klassen- und elitentheoretisch orientierter Diskussion wurde auch Staniszkis Begriff des politischen Kapitalismus elitentheretisch umformuliert und neutralisiert (Szalai 1994, Szelenyj/Szelenyj 1995). 


\section{Globaler Kapitalismus, die Effizienz von politischen Institutionen und soziale Integration - zur Konzeptualisierung und Abgrenzung des »politischen Kapitalismus«}

Wenn wir oben unter Verweis auf Thomas und North die institutionelle Innovation von »Eigentumsrechten « als Beginn der Differenzierung von Staat und Gesellschaft gefaßt und gefordert haben, diesen Gesamtzusammenhang zum normativen Bezugspunkt der Analyse der ökonomischen Transformationen zu machen, so heißt das natürlich nicht, daß die postsozialistische Entwicklung umstandslos am Modell der sich bis zum 19. Jahrhundert konstituierenden bürgerlichen Gesellschaft zu messen sei. Die klassische bürgerliche Gesellschaft, als ein durch das staatliche Gewaltmonopol befriedeter und durch staatlich sanktionierte Institutionen geregelter Raum einer gesellschaftlich erweiterten ökonomischen und politischen Interaktion von Privatleuten, ist sicherlich ein unzulängliches Modell des demokratischen Kapitalismus unter Bedingungen des 20. Jahrhunderts, in denen der Raum der bürgerlichen Gesellschaft durch einen organisierten Kapitalismus und eine in ökonomischer und politischer Dimension inkorporierte Gesellschaft vermachtet ist.

Hat so David Stark nicht doch Recht, wenn er die institutionellen Erscheinungsformen des post-sozialistischen politischen Kapitalismus mit denen hoch organisierter und kooperativ verflochtener spät-moderner Wirtschaftsaktuere gleichsetzt? Sind nicht auch für erfolgreich in der globalen Wirtschaft sich behauptende Wirtschaftsakteure die Einbettung in eine komplexe staatlich hergestellte Infrastruktur und die Verflechtung ökonomischer und politischer Macht charakteristisch?

In der Diskussion um die aktuelle und künftige Rolle des Staates hat sich seit den achtziger Jahren ein Paradigmenwechsel vollzogen. Dem liegt die Einsicht zu Grunde, daß mit der Internationalisierung der Kapitalmärkte und der Globalisierung des Wettbewerbs die Voraussetzungen für eine nationale staatliche Wirtschaftspolitik - im Sinne keynesianischer Konjunktursteuerung - entfallen und die autonomen Handlungsmöglichkeiten auf staatlicher Ebene zunehmend eingeschränkt werden. Die neue, sich in den achtziger Jahren durchsetzende Thematisierung des Staates, fragte weniger nach dessen internen regulativen Funktionen, sondern nach der vermittelnden Rolle des Staates für die Interaktion gesellschaftlicher Akteure in einer offenen, zunehmend entgrenzten, globalen Wirtschaft. Unter solch einer Fragestellung wurden in der neueren westlichen Debatte die politischen und gesellschaftlichen Einbettungen der Unternehmenstätigkeit in staatliche Institutionen und kulturelle Kontexte als wichtige und in ihrer Bedeutung zunehmende Aspekte von Wettbewerbs- und Entwicklungsvorteilen thematisiert. 
In seiner 1990 veröffentlichten Studie hat Porter (vgl. auch Messner/ Meyer-Stamer 1993) auf die Einbettung von Unternehmen in einen institutionellen Kontext und die produktivitätssteigernde Leistung von Netzwerken zwischen Unternehmen sowie zwischen diesen und staatlichen oder nichtstaatlichen Dienstleistungsträgern aufmerksam gemacht. In ähnlicher Weise haben Studien zu neuen Industriedistrikten in Dänemark, BadenWürttemberg und Norditalien auf neue Formen regionaler Kooperation verwiesen, die auf arbeitsteilige Spezialisierung sowie die Integration bestimmter Funktionen der Unternehmen (wie der Absatz auf entfernten Märkten) und die Entwicklung einer gemeinsamen Infrastruktur (Ausbildung, Informationssysteme) hinausliefen. In beiden Fällen resultierten die konstatierten Wettbewerbsvorteile aus Kooperation. Die von Porter beschriebenen cluster beziehen sich dabei vor allem auf hoch entwickelte Industriebranchen, wissenschaftliche Einrichtungen und die Transfers und das Pooling von spezialisiertem technischen Know How. Im Falle der in den Industrial district-Ansätzen beschriebenen Kooperationsformen waren die Akteure lokale Klein- und Mittelbetriebe sowie lokale politische Einrichtungen. Produktivitätsgewinne resultierten hier gewissermaßen aus der Kooperation und Kooperationsfähigkeit selber. Wichtig ist dabei aber festzuhalten, daß beide Ansätze zwar auf eine auch informelle Kooperation von staatlichen und privatwirtschaftlichen Akteuren und eine Verdichtung der Kontakte verweisen, diese Muster aber in einem hoch kompetitiven Umfeld ansiedeln und die Verdichtung der Kooperation nicht mit einer Entdifferenzierung politischer und ökonomischer Institutionen zu verwechseln ist.

Lassen sich die über den »politischen Kapitalismus « gesicherten »Wettbewerbsvorteile « mit den von Porter und den Industrial district-Studien angesprochenen Ressourcen vergleichen? David Stark selber hat darauf verwiesen, daß die formalen Kreuzungen von staatlichem und privatem Eigentum sowie die personelle Vernetzung von Politik und Ökonomie mit einer Umgestaltung des Staates in eine »Schuldenverwaltungszentrale« einhergehe (Stark 1994, 1996). Das aber heißt, daß das Ergebnis der »Rekombination« von Eigentum nicht mit den von Porter und den Industrial-district Studien hervorgehobenen neuen Kooperationsformen gleichgesetzt werden kann.

Die ökonomische Funktion des »politischen Kapitalismus« ist nicht die Produktivitätssteigerung durch technische Innovation und Kooperation. Die »Wettbewerbsvorteile« der post-sozialistischen Akteure und Netzwerke beruhen vielmehr auf exklusiven Möglichkeiten des Zugangs zu Informationen und Finanzmitteln, auf politischen Privilegien also und darüber gesicherten Möglichkeiten der Externalisierung von Kosten an die Gesellschaft. Im »politischen Kapitalismus « reproduziert sich somit ein institutionelles Grundmuster der sozialistischen Ökonomie. Deren grundlegende Merkmale waren eine politische Umverteilungswirtschaft und die Formel 
vom staatlichen Eigentum. Diese Formel stand für eine für die sozialistische politische Ökonomie charakteristische Fragmentierung und Diffusion von Eigentumsrechten. Denn das formal staatliche Eigentum wurde über die staatliche Verwaltung als Hierachie von Dispositionsspielräumen realisiert, die in einem von Land zu Land unterschiedlichem Maße bürokratisch verregelt, allesamt aber der Prärogative und der Kaderpolitik (Nomenklatur) des politischen Machtzentrums untergeordet waren. D.h. über den Apparat der Produktionsverwaltung waren formelle und informelle Zugriffsmöglichkeiten auf das Staatsvermögen - unterschiedlich und in einer von Land zu Land wechselnden Weise verteilt. Die so definierten Verfügungsrechte waren in dem durch die Nomenklatur und die zentrale Umverteilung zusammengebundenen Apparaten aber nicht als persönliche Verantwortlichkeit konstitutierende Eigentumsrechte institutionalisiert. Der "politische Kapitalismus « verweist auf Privatisierungsmechanismen und Formen von privatem Eigentum, die über die Aktivierung eines entformalisierten politischen Kapitals »schwache « Eigentumsrechte und tradierte Orientierungen auf staatliche Renten institutionell reproduzieren. Die »Wettbewerbsvorteile«, die sich aus solchen Institutionen ergeben, beruhen nicht auf Produktivitätsgewinnen durch Innovation und Kooperation, sondern auf der Externalisierung von Kosten an den Staat und die Gesellschaft.

Mit anderen Worten: Die Funktion mehr oder weniger institutionalisierter Formen der Kooperation und Vernetzung von Wirtschaftsunternehmen sowie von ökonomischen und politischen Akteuren läßt sich nicht schon aus den kooperativen Organisationsformen selber ableiten. Moderne Wettbewerbsfähigkeit ist nicht nur Resultat der Einbettung von Unternehmen in kooperative Netzwerke und eine entwickelte Infrastruktur. Die Funktion der Netzwerke und die Leistungen der Infrastruktur sind im Kontext der Leistungsfähigkeit der politischen Institutionen zu sehen.

In seinem 1993 veröffentlichten Buch synthetisiert Robert D. Putnam eine Vielzahl von empirischen Untersuchungen über die Funktionsweise der regionalen und lokalen Selbstverwaltungen in Italien als Studie über die Effizienz von demokratischen Institutionen und die Angewiesenheit effizienter politischer Institutionen auf ihre gesellschaftliche und kulturelle Einbettung. Die Thesen Putnams lassen sich dahingehend zusammenfassen, daß ökonomische Entwicklung auf effiziente politische Institutionen und diese wiederum auf lebendige zivile Gemeinschaften angewiesen sind.

Diese Arbeit kann im Zusammenhang unserer Argumentation als Explikation der »anderen « politisch-gesellschaftlichen Seite der Thesen von Porter bzw. der Industriedistrikt-Studien betrachtet werden. Zum einen verschiebt Putnam die Perspektive insofern, als er zum Bezugspunkt der Frage nach »Wettbewerbsfähigkeit « weder das Unternehmen noch das Netzwerk von Unternehmen macht, sondern die lokale Gesellschaft. Zum anderen können 
wir Putnams Buch aber auch als historische »Verlängerung« der Studien zur Bedeutung der Institutionalisierung von Eigentumsrechten für Wirtschaftswachstum lesen, weil er nach Bedingungen der Effizienz von demokratischen politischen Institutionen im Kontext verdichteter Beziehungen zwischen Politik und Ökonomie fragt. Mit der zunehmenden Dichte der Interaktionsbeziehungen steigt die ökonomische Bedeutung der Transaktionskosten (North 1990) der wirtschaftlichen Tätigkeit. Deren Höhe sinkt in dem Maße, in dem demokratische staatliche Institutionen und damit Eigentumsrechte effizient institutionalisiert sind, d.h. um so weniger ihre Geltung auf staatliche Gewaltandrohung angewiesen und durch die kulturelle Verankerung in zivilen Gemeinschaften informell gesichert ist.

In ähnlicher Weise wie North am Beispiel des Verhältnisses von Staat und Gesellschaft in England und Spanien unterschiedliche, politisch definierte Entwicklungspfade kontrastiert hatte, zeigt Putnam, wie sich - in scharfem Kontrast zum Süden des Landes - in Norditalien Institutionen der zivilen Stadtkultur in gewandelter Form und auf informeller Ebene - auch unter Jahrhunderten autokratischer Herrschaft - reproduziert und als zivilgesellschaftliche Kompetenzen der regionalen Gesellschaften die Effizienz der politischen Institutionen und darüber die ökonomischen Wettbewerbsvorteile in der Nachkriegsentwicklung bestimmt haben. Mit dem Konzept der »zivilen Gemeinschaft« wird das institutionelle Muster der Zivilgesellschaft in Kategorien kultureller Kompetenzen übersetzt. Dabei kontrastiert Putnam das Syndrom der »zivilen Gemeinschaft« dem eines mafiosen Klientelismus. Die zivile Gemeinschaft charakterisiert sich durch bürgerliches Engagement, das sich an der Idee eines vorgestellten (abstrakten) Gemeinwohls orientiert, die Bürger als prinzipiell Gleiche und ihre Beziehungen als Institutionen denkt und sich als Solidarität, Vertrauen und Toleranz über horizontale Assoziationen realisiert und reproduziert. Dem wird ein »amoralischer Familiarismus« (Banfield) entgegengestellt, der Sozialbeziehungen als persönliche Abhängigkeiten zwischen Patron und Klient, Chef und Petent hierarchisch und konkret denkt und sich als ein Opportunismus artikuliert, dessen Grundlage im fehlenden Vertrauen in die Gegenseitigkeit von Beziehungen und Verpflichtungen gesehen wird. Es sind nach Putnam die »moralische Ressource« des Gemeinsinns (Hirschmann) einerseits und der Umfang des »Opportunismus « andererseits, die die Effizienz von politischen Institutionen und die Höhe ökonomischer Transaktionskosten bestimmen.

Der Begriff des »amoralischen Familiarismus« wurde von Banfield (1958) entwickelt. Seine Studie über ein süditalienisches Dorf zeigte eine auf den engen Kreis der Kernfamilie beschränkte Sozietät. Alle über die Familie hinausgehenden Kontakte wurden durch fehlendes Vertrauen und fehlendes Verantwortungsgefühl geprägt. Größere soziale Einheiten - Nachbarschaft, 
Dorf, Kirche, Nation - weckten nicht das Gefühl der Identifikation. Mit anderen Worten: ein wie auch immer definierter gesellschaftlicher Horizont und damit abstrakte soziale Normen fehlten. Im »amoralischen Familiarismus « sehen Banfield und mit ihm Putnam die anthropologischen Grundlagen einer als »Mafia« organisierten Quasi-Gesellschaftlichkeit. Deren Merkmal ist nicht nur die hierarchische Struktur und der persönlich konkrete Charakter der Abhängigkeit, sondern die Gewaltförmigkeit von Verhältnissen, die angesichts des Fehlens normativer Standardisierung allein durch Opportunismus und das Faustrecht geordnet werden.

Im politischen Leben schlagen sich diese divergierenden Muster als Orientierung auf politische Programmatiken und allgemeine politisch-rechtliche Regeln des Zusammenlebens bzw.eine Orientierung auf Personen (nicht als Symbole für abstrakte Ideen sondern) als unmittelbare Hebel zur Durchsetzung persönlicher Interessen (Posititionen, Lizenzen) nieder. Das erste Politikmuster demokratischer Interessenvermittlung geht einher mit hoher Transparenz, relativ egalitären Chancen und abgeflachten sozialen Distanzen, das zweite Muster einer klientelistischen Interessendurchsetzung ist mit der Undurchsichtigkeit politischer Entscheidungsprozesse und einer elitären und in hohem Maße hierarchischen Sozialstruktur verbunden.

Es ist dabei in Hinblick auf Osteuropa interessant, daß der Kern der Polarisierung des Begriffspaares »amoralischer Familiarismus « und »zivile Gemeinschaft« sich nicht auf die bloßen politischen Beziehungsmuster, d.h. vertikaler Klientelismus versus horizontale Organisation in Verbänden bezieht. Putnam verweist sogar darauf, dass klientelistische Politikmuster in Norditalien auch als Hebel für eine zivilgesellschaftliche Interessenpolitik genutzt wurden, indem darüber soziale (etwa regionale oder lokale) Partikularinteressen artikuliert wurden.

Im Zusammenhang unserer Frage nach der Bewertung der Phänomene des "politischen Kapitalismus « ist an dieser Stelle festzuhalten, daß das »soziale Kapital« der zivilen Gemeinschaft sich keineswegs auf die Existenz von Netzwerken bezieht, sondern auf die moralische Ressource normativer Sozietät. Die post-kommunistischen Netzwerke können insofern als Erscheinungsform des genauen Gegenteils ziviler Gemeinschaften verstanden werden, als sie sich - in der institutionellen Nachfolge der Nomenklatur auf persönliche Loyalität und Opportunismus gründen und die Durchsetzung persönlicher Interessen zum Ziel haben.

Dennoch kann - was die moralischen Ressourcen der post-sozialistischen Gesellschaften betrifft - weder von einem schlichten Gegensatz zwischen korrumpierten Machteliten und integrer Gesellschaft ausgegangen werden, noch scheint das bipolare Begriffspaar »amoralischer Familiarismus« und »zivile Gemeinschaft« hinreichend zur Beschreibung der Integrationsmuster der sozialistischen Gesellschaften zu sein. 
Die polnischen Soziologen Tarkowska und Tarkowski haben in den 80er Jahren gezeigt, daß bäuerliche Familien andere Standards gegenüber »eigenen Leuten « und gegenüber »Fremden« anwandten. Im Anschluß an Banfield konstatieren auch sie eine Verengung des gesellschaftlichen Raumes und interpretieren dies als Ausdruck sozialer Desintegration (Tarkowska/ Tarkowski 1990). Studien in anderen Milieus zeigten, familiaristische $\mathrm{Mu}-$ ster in allen sozialen Schichten, wobei sich der Kreis der über Netzwerke vermittelten sozialen Kontakte mit dem Bildungsniveau und der Schichtzugehörigkeit erhöhte. Als am stärksten atomisiert erwies sich die Kategorie der unqualifizierten Arbeiter bäuerlicher Herkunft, deren Kontakt zum Dorf zerbrochen und nicht durch neue Bindungen im städtischen Milieu ersetzt war. Diese Kategorie verweist zugleich auf die stalinistische Vorgeschichte, als die massenhafte Zuwanderung von bäuerlichen Arbeitern die Arbeitermilieus zersetzte. Über die am meisten ausgeweiteten Kontakte verfügte die akademisch gebildete Intelligenz, wobei die qualifizierten Arbeiter der zweiten Generation eine Zwischenposition einnahmen, sich aber dem Lebensstil der Intelligenz mit der Orientierung auf gesellschaftliche Kontakte angeglichen hatten (Markarczyk 1978, Wnuk-Lipinski 1985). In einer kulturanthropologischen Studie hat Narojek biographische Texte auf die symbolische Konstruktion von sozialen Beziehungen analysiert und das zugrundeliegende Gesellschaftsbild herausdestilliert (Narojek 1982). Die Studie zeigt eine gespaltene Realitätswahrnehmung: Strukturell gleiche Situationen werden binär typisiert, je nach dem, ob sich der Sprecher als Teilnehmer oder Ausgeschlossener erfährt. Narojek hat diese Wahrnehmungsmuster als Ausdruck einer »stammesgesellschaftlichen« Integration der sozialistischen Gesellschaft interpretiert.

In Analogie zu Banfields Formulierung könnte man von einem »amoralischen Tribalismus « insofern sprechen, als wir es mit einer fragmentierten Moral zu tun haben. Es entsteht kein einheitlich normativ integrierter gesellschaftlicher Raum, wohl aber ein Geflecht von Netzwerken, die intern nach moralischen Standards integriert sind und »soziales Kapital« (Coleman) schaffen, deren Außenkontakte aber durch Opportunismus geprägt sind. ${ }^{7}$ Im Zusammenhang der hier angestellten Überlegungen scheint eine schärfere begriffliche Unterscheidung nützlich. Die Reduktion von Sozietät auf den Kern der Familie - im Sinne des von Banfield beobachteten »amoralischen Familiarismus«, bedeutet, daß wir es mit einer anomischen Ordnung insofern zu tun haben, als die Beziehungen in der traditionalen Familie nicht normativ, sondern durch Status (Patriarchat) und personenbezoge-

7 Solche Integrationsmuster sind nicht nur für oppositionelle oder traditionale private Kreise charakteristisch, sie betreffen auch die Milieus unterschiedlicher Segmente der Machteliten, deren Netzwerke in vielen Fällen - wie bei anderen Gruppen auch - auf die gemeinsame Ausbildungszeit verweisen. 
ne Verpflichtungen (Verwandschaft) stablisiert werden. Zugehörigkeitsgefühle basieren hier nicht auf geteilten Werten und Normen, sondern auf Status und emotionaler Bindung. Bei den oben angesprochenen Formen des »amoralischen Tribalismus « handelt es sich dagegen um Phänomene fragmentierter Moral und nicht der Anomie. Diese begriffliche Schärfung bietet auch ein Unterscheidungskriterium von »Mafia (als anomische, gewaltförmige und personenbezogene Abhängigkeitskette) und informellen, durch die Normen von Milieus integrierten Netzwerken: im Unterschied zur Mafia produzieren die beschriebenen Netzwerke soziales Kapital.

In der Dimension der politischen Ordnung definiert das Muster des »amoralischen Familiarismus « die Auflösung des Gewaltmonopols in einem kriminellen Staat oder einem offen anarchischen Bandenwesen. Die fragmentierte Moral neo-tribalistischer Integration ist verbunden mit einer Personalisierung sozialer Beziehungen bzw. der kognitiven Systematisierung der sozialen Umwelt nach binären Mustern. Solche Mentalitäten können als »zivilisatorische Inkompetenzen « (Sztompka 1993, 1994) begriffen werden, die einer Realisierung rechtsstaatlicher und zivilgesellschaftlicher Sozialbeziehungen entgegenstehen und exklusiven Cliquenbildungen und klientelistischen Politikmustern förderlich sind.

Nach dem Grad der Demoralisierung und Gewaltförmigkeit sozialer Beziehungen und der kulturellen Fragmentierung lassen sich die mittel- und osteuropäische Gesellschaften unterscheiden. Auch innerhalb der Gesellschaften lassen sich unterschiedliche Integrationsformen ausmachen. Historisch ist das Muster des »amoralischen Familiarismus« dabei sowohl innerhalb unterschiedlicher Segmente des Apparates (insbesondere der politischen und Gewaltapparate) als auch in mit diesen nicht selten kooperierenden kriminellen Milieus herausgebildet worden. Kulturell integrierte Beziehungsnetze bildeten die politischen und kulturellen Oppositionsbewegungen, intellektuelle und traditionale Milieus sowie lokale Gemeinschaften heraus, die von der sozialistischen Migration weniger betroffen waren. Aber auch in den verschiedenen Gruppierungen der professionellen Nomenklatur funktionierten normative Standards. Darüber hinaus hatten sich auch unter sozialistischen Bedingungen in unterschiedlichem Umfang Interaktionsformen herausgebildet, die das Muster des »amoralischen Tribalismus« sprengten. Ich meine hiermit nicht die »zweite Wirtschaft« als »Gegengesellschaft« (Hankiss 1991), weil diese eher ein Übungsfeld für opportunistische Atomisierung gewesen sein dürfte. ${ }^{8}$ Ich meine hiermit einerseits

8 Es scheint problematisch, die »zweite Ökonomie« als Experimentierfeld marktkonformen Verhaltens zu betrachten. Zum einen waren die Wirtschaftsakteure in den Nischen der Staatswirtschaft auf diese und das Wohlwollen der Verwaltung angewiesen und so in Machtbeziehungen verankert. Zum anderen war die zweite Wirtschaft, selbst da, wo sie, wie in Ungarn und Polen, weit legalisiert wurde, durch »graue«, kriminelle Verhaltensweisen charakterisiert. Die Idealisierung insbesondere der ungarischen »zweiten Wirt- 
soziale Bewegungen - wie die Solidarnosc oder die Sajudis -, deren moralischer Fundamentalismus oder nationales Ethos die kulturelle Segmentierung der Gesellschaft auf symbolischer Ebene dadurch aufhob, daß sie die Vorstellung eines abstrakten allgemeinen Wohls erschufen. Und ich denke andererseits an unterschiedliche Formen der zivilen Selbstorganisation, wie die »gesellschaftliche « Organisation von Einkaufsschlangen, die Organisation »gesellschaftlicher « Wachen an den Parkplätzen der Wohnsiedlungen oder die informelle Herausbildung gesellschaftlicher Funktionen wie Schrebergärtnergemeinschaften. ${ }^{10}$

Bevor wir uns der ländervergleichenden Skizze zuwenden, sind die bisherigen Überlegungen zusammenzufassen. Ausgehend von der Annahme einer politischen Bestimmung der Wirtschaftsverfassung über den Charakter des Staates und der sozialen Integration der Gesellschaft lassen sich der »amoralische Familiarismus « einer mafiosen Ökonomie zuordnen und zivile Kompetenzen und Gemeinschaften als Innenfutter eines demokratischen Kapitalismus begreifen.

Schwieriger erscheint die Bewertung der fragmentierten Moral tribalistischer Netzwerkstrukturen. Zum einen haben wir es nicht mit einem klar definierten Idealtypus zu tun, sondern mit einer ganzen Spannbreite von Beziehungen, die sich je nach Reichweite und inhaltlichen Merkmalen der normativen Integration in ihren politischen Funktionen unterscheiden. Zum anderen haben normativ integrierte Netzwerke für unterschiedliche Formen der Wirtschaftsorganisation eine andere Funktion.

So hat Francis Fukuyama den »Familiarismus " ${ }^{11}$ als moralische Ressource für ein flexibles Kleinunternehmertum in Norditalien oder der chinesischen Diaspora ausgemacht (Fukuyama 1995). Auch für den Aufstieg des Kleinstunternehmertums in Polen, das von den Dienstleistungen des Bankensektors abgeschnitten war, bildeten die private Hilfe und der Rückhalt von Verwandten und Freunden eine ökonomische Voraussetzung. Andererseits zeigt die Dominanz etwa der Vietnamesen in bestimmten Bereichen der

schaft« zur Bürgergesellschaft mit der Figur des »Bourgeois« übersah, daß in der bürgerlichen Gesellschaft der »bourgeois« - um als solcher zu handeln - auf den »citoyen« angewiesen war, d.h. nur als »citoyen« auch »bourgeois« sein konnte.

9 Dies beruhte darauf, daß die Schlange ein Komitee bildete, das die Namen der Wartenden notierte und in festgesetzten Zeiträumen die Zusammensetzung der Schlange überprüfte, sodaß die Betroffenen nicht mehr mehrere Tage vor einem Geschäft mit Möbeln oder Kühlschränken verbringen, sondern sich nur noch in regelmäßigen Abständen melden mußten.

10 Auf gemeinsame Initiative der Mitglieder solcher Siedlungen wurden soziale Dienste und soziale Einrchtungen wie etwa ein Klubhaus geschaffen; vgl. die Beschreibung dieser kleinbürgerlich geordneten Welt in den Erzählungen von Bohumil Hrabal wie z.B. »Schneeglöckchenfeste«.

11 Fukuyama faßt hierunter nicht den amoralischen Familiarismus der Mafia, sondern Sozietätsformen, die dem oben beschriebenen Muster einer tribalistisch fragmentierten Moral entsprechen. 
post-sozialistischen Basarökonomie nicht nur das soziale Kapital ethnischer Gemeinschaften, sondern auch deren exklusiven Charakter. Fukuyama hat zudem darauf verwiesen, daß familiaristische Integrationsmuster die formale Rationalität verbandsförmiger Organisation aushöhlen und einer Stabilisierung bürokratischer Großunternehmen mit effizienter corporate governance wenig förderlich sind. Bereits oben hatten wir die personelle Vernetzung von neuen politischen Institutionen und ökonomischen Akteuren im post-sozialistischen Kontext problematisiert, weil sie eine anzustrebende institutionelle Differenzierung von Politik und Ökonomie unterläuft und von opportunistischen Haltungen getragen wird. Wenn wir familiaristische Orientierungen und die fragmentierte Moral tibalistischer Sozietät dem Organisationstyp des politischen Kapitalismus zuordnen, definieren wir diesen weniger eindeutig.

Wir können uns den »politischen Kapitalismus « als einen gesellschaftlichen Organisationstypus denken, dessen spezifische Ausformungen sich auf einem Kontinuum zwischen polarisierten Idealtypen eines $»$ kriminellen Kapitalismus « und eines »demokratischen Kapitalismus « verorten lassen. Der Konstruktion der Begriffe liegt die Annahme zugrunde, daß die Art und Weise der Transformation des Staates - und damit des Typus der neu sich konstituierenden politischen Macht - für die ökonomische Funktionsweise der neuen Ordnung von entscheidender Bedeutung und die Effizienz staatlicher Institutionen in hohem Maße Resultat der kulturellen Integrations- und Perzeptionsmuster ist. Mit anderen Worten: die moralischen Ressourcen und sozialkognitiven Kompetenzen von Gesellschaften werden als mittelbar determinierende Faktoren der Diversifikation von ökonomischen Beziehungen betrachtet. Diese Determination ist zunächst eine begriffliche und keine historische.

Als idealtypische Konstruktionen sind sowohl der kriminelle Kapitalismus als auch der demokratische Kapitalismus Abstraktionen und nicht Bilder historischer Wirklichkeit. Eine Wirtschaftsordnung ohne jegliche moralische Ressourcen, die allein der Extraktion und der Reproduktion gewaltförmiger Macht dient, läßt sich kaum als soziale Realität vorstellen. Dasselbe gilt für einen ökonomischen Mechanismus, der in eine normativ integrierte und demokratisch geordnete Gesellschaft eingebettet und dessen ökonomischer Inhalt die Produktivitätserweiterung ist. Historische Figurationen treten als Gemisch mit unterschiedlichen Gewichtsverteilungen auf. Das Schema formuliert so nicht mehr als Bezugsmodelle für die Analyse historisch konkreter Gesellschaften. 
Politischer Kapitalismus

\begin{tabular}{|c|c|c|c|}
\hline & $\begin{array}{l}\text { Krimineller } \\
\text { Kapitalismus } \\
\end{array}$ & & $\begin{array}{l}\text { Demokratischer } \\
\text { Kapitalismus }\end{array}$ \\
\hline $\begin{array}{l}\text { Ökono- } \\
\text { mie }\end{array}$ & $\begin{array}{l}\text { Extraktion } \\
\text { Umverteilung } \\
\text { »Schutzgeld«, } \\
\text { gewaltförmig orga- } \\
\text { nisierte u. kontrol- } \\
\text { lierte mafiose } \\
\text { »Märkte« }\end{array}$ & $\begin{array}{l}\text { Extraktion/ Produktion, } \\
\text { Externalisierung von } \\
\text { Kosten, } \\
\text { rent-seeking, } \\
\text { Monopolisierung von } \\
\text { Märkten }\end{array}$ & $\begin{array}{l}\text { Produktion, } \\
\text { Produktivitäts- } \\
\text { steigerung, } \\
\text { profit-seeking, } \\
\text { Kompetitivität von } \\
\text { Märkten }\end{array}$ \\
\hline Staat & $\begin{array}{l}\text { Anarchie/ } \\
\text { Faustrecht } \\
\text { (keine Eigentums- } \\
\text { rechte, keine } \\
\text { Vertragsdurch- } \\
\text { setzung) }\end{array}$ & $\begin{array}{l}\text { fragmentiertes und durch- } \\
\text { löchertes Gewaltmonopol, } \\
\text { schwacher Rechtsstaat, } \\
\text { schwache Eigentumsrechte, } \\
\text { schwache Geltung von Ver- } \\
\text { trägen }\end{array}$ & $\begin{array}{l}\text { Gewaltmonopol } \\
\text { Rechtsstaat } \\
\text { starke Eigentumsrechte } \\
\text { Geltung von } \\
\text { Verträgen }\end{array}$ \\
\hline Politik & & Oligarchie & $\begin{array}{l}\text { Demokratische } \\
\text { Institutionen }\end{array}$ \\
\hline $\begin{array}{l}\text { Gesell- } \\
\text { schaft }\end{array}$ & $\begin{array}{l}\text { Atomisierung, } \\
\text { gesellschaftliches } \\
\text { Vakuum }\end{array}$ & $\begin{array}{l}\text { Patronagepolitik oligarchi- } \\
\text { scher Parteiapparate, die in } \\
\text { einer »hybriden« Wirtschaft } \\
\text { verankert sind, } \\
\text { Klientelismus als Form der } \\
\text { Interessenartikulation, } \\
\text { schwache Öffentlichkeit, } \\
\text { geringeTransparenz der } \\
\text { Politik }\end{array}$ & $\begin{array}{l}\text { Vermittlungsfunktion } \\
\text { von Parteien und Ver- } \\
\text { bänden, die in der differen- } \\
\text { zierten Gesellschaft veran- } \\
\text { kert sind, } \\
\text { zivilgesellschaftliche } \\
\text { Aktivität, } \\
\text { starke Öffentlichkeit, } \\
\text { Transparenz der Politik }\end{array}$ \\
\hline $\begin{array}{l}\text { morali- } \\
\text { sche Res- } \\
\text { sourcen }\end{array}$ & $\begin{array}{l}\text { Anomie } \\
\text { amoralischer } \\
\text { Familiarismus, } \\
\text { Opportunismus }\end{array}$ & $\begin{array}{l}\text { tribalistisch fragmentierte } \\
\text { Moral, Personalisierung } \\
\text { sozialer Beziehungen, } \\
\text { konkrete Gemeinschaft, } \\
\text { ambivalente moralische } \\
\text { Ressourcen, Opportunismus }\end{array}$ & $\begin{array}{l}\text { civic community } \\
\text { generalisiertes Vertrauen, } \\
\text { abstrakte Gemeinschaft, } \\
\text { starke moralische } \\
\text { Ressourcen, } \\
\text { geringer Opportunismus }\end{array}$ \\
\hline
\end{tabular}




\section{Unterschiedliche Transformationspfade}

Das idealtypische Konstrukt gibt uns ein Instrument zur paradigmatischen Neuformulierung von $»$ Transformation « als institutioneller Anpassung: Die Revolutionen in Osteuropa können als Bruch von drei für das sozialistische System konstitutiven Axiomen verstanden werden. Die politische Prärogative der Partei (institutionalisiert als Nomenklatur) wurde durch die Einführung demokratischer Mechanismen und die Differenzierung des politischen Systems beseitigt; das Tabu des staatlichen Eigentums wurde aufgehoben, private Eigentumstitel zugelassen und die politische Abschließung der sozialistischen Ökonomien zur Disposition gestellt. Demokratisierung, Privatisierung und Öffnung sind zugleich komplementäre Systembrüche, insofern als Demokratisierung Voraussetzung für die Durchsetzung von Eigentumsrechten und Privatisierung Bedingung einer strukturellen Öffnung der Wirtschaft ist. Dieser formale Bruch stellt die Akteure gewissermaßen in eine neue Welt, definiert neue Handlungs- und Erfolgsbedingungen. Der Begriff des politischen Kapitalismus soll in diesem Kontext helfen, die Art und Weise der Anpassung und den Charakter des institutionellen Wandels zu beschreiben. Er dient dabei als ein Instrument zur Analyse der Kontinuität im Wandel. Der Begriff des politischen Kapitalismus bezieht sich auf den Umfang, in dem die Logik der Reproduktion von Macht in die neue kapitalistische Ordnung eingebaut bleibt, zum Hebel der Transformation und zum Bestimmungsfaktor der neuen Gesellschaftsstruktur wird.

Phänomene des politischen Kapitalismus lassen sich in allen post-sozialistischen Gesellschaften ausmachen. Dennoch unterscheiden sich die Entwicklungen nach der Richtung des institutionellen Wandels deutlich. Das am Ende dieses Abschnitts vorgestellte Entwicklungsschema soll im Folgenden durch Skizzen der länderspezifische Besonderheiten des politischen Kapitalismus illustriert werden.

Schon in der zweiten Hälfte der achtziger Jahre schufen eine Reihe gesetzlicher Neuregelungen in Polen wie in Ungarn die Voraussetzungen für eine »spontane Privatisierung «. Die weiche Finanzierung der staatlichen Betriebe und die formelle und/oder personelle Vernetzung von staatlichen Akteuren und privater Geschäftswelt eröffneten verschiedene Formen des rentseeking und damit des Transfers von staatlichen Ressourcen in private Hände (Staniszkis 1991, 31-68).

In Polen wurden diese informellen Formen des Transfers nach 1989 unter den Regierungen der Solidarnosc-Eliten blockiert. Die Privatisierung wurde einer relativ weitgehenden politischen Kontrolle unterworfen. Dies war möglich, weil die neue Regierung aus dem symbolischen Reformkonsens breiter Teile der Bevölkerung Handlungsmacht und Autonomie gegenüber etablierten administrativen Interessen gewinnen konnte (Tatur 1995). Zudem war die Privatisierung in Polen ein konflikthafter Prozeß, an dem auch 
die Belegschaften bzw. deren Interessenvertretungen (Räte und Gewerkschaften) teilnahmen (Kloc 1996). Das mag in Mutterbetrieben der Gewerkschaft in traditionalen Branchen auch ein Hindernis rascher Restrukturierung gewesen sein, ${ }^{12}$ die Existenz konflikthafter Machtzentren bildete aber zugleich auch ein Instrument der Kontrolle des Managements und ein Sensorium für konflikthafte Anpassungsprozesse. Auf politischer und betrieblicher Ebene bildeten symbolischer Konsens und Konflikt in dieser Phase die für Polen charakteristische Methode des institutionellen Wandels (Deppe/Tatur 1995, 1996). Zum quantitativ dominierenden Privatisierungspfad wurde die »Liquidation«, wobei Teilbetriebe an Außenstehende oder die Belegschaften verkauft oder in Zahlung gegeben wurden. Die Besonderheit dieser Form von Insider-Übernahmen war nicht nur, daß das Belegschaftseigentum auch Mitglieder der industriellen Mittelschicht zu Nutznießern der Umgestaltung machte, sondern daß die neuen Betriebe von der alten, liquidierten Struktur unabhängig wurden. Das schon 1992 einsetztende industrielle Wachstum wurde von einem expandierenden Privatsektor getragen, der als Konsequenz dieser Privatisierungspolitik und der »monetaristischen « Härte der Finanzpolitik in hohem Maße von staatlicher Zufinanzierung abgenabelt war. Die Lösung der privaten wie der kommerzialisierten staatlichen Unternehmen vom Staat sollte das rent-seeking blockieren und die strukturelle Anpassung als einen Prozeß »produktiver Zerstörung « (Schumpeter) erzwingen. Der »spontane« Transfer politischer Macht in ökonomisches Kapital war in Polen so nach 1990 zunächst in hohem Maße in den Bereich illegaler oder halblegaler »Affairen« im Außenhandel und dem Bankwesen abgedrängt. ${ }^{13}$

Schon von den Solidarnosc-Eliten wurde das liberale Konzept einer nur spontanen strukturellen Anpassung seit 1992 problematisiert und die Notwendigkeit der Schaffung strategischer Akteure gesehen. Noch unter dem liberalen Privatisierungsminister Lewandowski wurden Holdinggesellschaf-

12 Als Beispiele für eine konservative Rolle der Gewerkschaften werden die Danziger Werft, das Traktorenwerk Ursus und der Kohlebergbau zitiert, wo neben anderen Faktoren starke Gewerkschaften eine schnelle Strukturanpassungspolitik verhinderten und unter neuen Bedingungen - sich ähnlich verhielten wie die Betriebsleitungen zuvor, d.h. abwartend und auf staatliche Hilfe hoffend.

13 Schlüssel solcher Praktiken waren in der Regel exklusive Informationen über Gesetzeslücken, etwa im Bankwesen (ein Beispiel war die Art-B-Affaire) oder im Bereich der Zollbestimmungen (Alkoholaffaire), die für formal legale Manipulationen und Betrügereien genutzt werden konnten. Eine anders gelagerte Geschichte war die FOZZ-Affaire. FOZZ war ein 1989 gebildeter Fonds, der heimlich die Schulden des polnischen Staates aufkaufen sollte, und dafür Mittel aus dem Staatshaushalt erhielt und große Teile davon - nach dem Urteil der Prokuratur - veruntreute. Es gab personelle Überschneidungen zwischen den Angeklagten in der FOZZ-Affaire und der Außenhandelszentrale Universal, die 1990 in eine Aktiengesellschaft umgestaltet wurde und sich in der Folgezeit zu einer der größten Kapitalgruppen Polens entwickelte. 
ten geschaffen, ${ }^{14}$ die Risiken und Kosten der Restrukturierung unternehmensintern abfedern sollten. Diese Holdings sollten als privatwirtschaftliche Akteure vom Staat unabhängig gemacht werden. Mit der Übernahme der Regierung durch die post-kommunistischen Parteien kam es 1993/94 im Bereich der Ordnungspolitik zu einem Strategiewechsel. Die neue Politik setzte nicht nur auf die systematische Schaffung von Holdings und eine gewisse Remonopolisierung, sondern auch auf die bewußte Schaffung hybrider Akteure und organisierter Märkte in zukunftsträchtigen Bereichen. Die neuen Hybriden waren um Außenhandels- und Finanzinstitutionen organisiert und nicht selten eng mit den post-kommunistischen Parteien verbunden. ${ }^{15}$ Zur Agenda des politischen Kapitalismus wurden nun Finanzeinrichtungen, deren Kreditvergabe der Staat in unterschiedlichem Umfang absicherte. Solche Garantien etwa an Handelsbanken sollten das von diesen bei der Kreditvergabe für Strukturanpassungs- und Markterweiterungspolitiken von Unternehmen übernommene Risiko reduzieren. Diese Politik war prinzipiell nicht kontrovers, sie bot aber neue Möglichkeiten der Einflußnahme im privaten oder parteipolitisch-klientelistischen Interesse. ${ }^{16}$ Mitte der neunziger Jahre schlug sich das anhaltend hohe Wirtschaftswachstum auch in einer Verbesserung der Lebensbedingungen und einem steigenden gesellschaftlichen Optimismus nieder. Erste Anzeichen von Krisenphänomenen, die seit dem Jahreswechsel 1996/97 in Polen sichtbar wurden, ${ }^{17}$ waren im Vorwahlkampf bagatellisiert und haben sich bislang

14 Das bekannteste Beispiel war die Neustrukturierung und Privatisierung der Stettiner Werft.

15 So war die schon erwähnte Gruppe Universal ebenso wie Elektrim mit der Sozialdemokratie (SdRP), die Agencja Rozwoju Gospodarczego mit der PSL verbunden. Universal besitzt Anteile an 50 weiteren Firmen, darunter dem privaten Fernsehsender Polsat. Die mit Universal verbundene Firma Ad Novum unterhält die Zeitung der SdRP »Tribuna« (vgl. Gazeta Wyborcza vom 5.5.1997).

16 Eine breite, auch große Teile der Bevölkerung einschließende Debatte über die staatliche Politik entzündete sich an der Liquidierung der Danziger Werft, der Wiege der Solidarnosc, wobei die Regierungsentscheidung Kreditgarantien für die engagierten Handelsbanken trotz vorhandener Aufträge für die Werft zu verweigern, mit anderen positiven Entscheidungen in ähnlich gelagerten Fällen, die ökonomisch weitaus weniger sinnvoll waren, konstrastiert wurde, wobei die Werftindustrie in Polen als entwicklungsfähige Branche gilt. Die fatale Situation der Danziger Werft 1997 wurde mit der politisch motivierten Liquidierungsentscheidung von 1988 (letzte kommunistische Regierung Rakowskis) und der selbstgewissen und verfehlten Politik von Direktion und Gewerkschaft zu Beginn der neunziger Jahre erklärt.

17 Polen wies wie andere mitteleuropäische Länder auch 1997 ein großes und steigendes Außenhandelsdefizit auf. Dieses Krisensymptom ist zwar mit einem anhaltenden Wirtschaftswachstum verbunden, der Importüberschuß wurde aber vor allem deshalb kritisiert, weil es sich neben relativ geringen Importen für den unmittelbaren Konsum vor allem um »Versorgungsimporte « für Reinvestitionen und die Weiterverarbeitung von Produkten für den inneren Markt handele. Zugleich wurden die Ressourcen des Privatsektors als gering, und die zwar steigende Investitionsrate als nach wie vor zu niedrig kritisiert. Ein besonderes Problem stellte die mit 19\% des BSP niedrige Sparquote und die Tatsache dar, daß die inländische Nachfrage durch steigende private Verschuldung aufgebläht 
nicht in einem Rückgang des hohen Wachstumstempos niedergeschlagen. ${ }^{18}$ In Ungarn hat ein vergleichbarer Bruch in der Transformationspolitik nicht stattgefunden. Hier markiert das Jahr 1990 keinen Einschnitt (so auch Kornai 1996). Ungeachtet ihrer anfänglichen Bemühungen gelang es der konservativen Regierung nicht, die politische Kontrolle und Initiative der Privatisierungspolitik in ihre Hände zu nehmen. Ungeachtet der formalen Kompetenzen der staatlichen Vermögensagentur und des formalen Rechts der Räte, Privatisierungsprozesse zu initiieren, blieb das Management auch nach 1990 der wichtigste Akteur im Umgestaltungsprozeß. Faktisch waren es die leitenden Manager, die den Privatisierungsprozeß initiierten und dominierten, auch wenn sie nun gezwungen waren, mit der staatlichen Vermögensverwaltung zu verhandeln. Das Scheitern des Versuchs, den Prozeß zu zentralisieren, stärkte die Macht des Managements weiter (Frydman u.a., 1993, 130f). In dieser Politik schlug sich nicht nur die Schwäche der konservativen Regierung, sondern auch das Fehlen einer betrieblichen Gegenmacht nieder. Denn ungeachtet der formalen Rechte der Räte, waren diese Organe im Kontext einer entpolitisierten Arbeiterschaft bedeutungslos. Die dominierende Form der Privatisierung war in Ungarn der Verkauf an Externe, was mit der außerordentlich großen Bedeutung ausländischer Investoren zusammenhängen dürfte, und »andere« Formen, eine Floskel, hinter der sich neben der Liquidierung auch die Übernahme durch Schuldner verbarg (World Bank 1996, 53). Das Ergebnis des Prozesses beschreibt Stark in seiner 1993 verfaßten Studie als »Rekombination« von Eigentum: privatwirtschaftlichen Satellitengesellschaften wurden um staatliche Mutterbetriebe herum ausgelagert und die Bildung von Holdinggesellschaften und Konglomeraten gefördert, wobei Eigentumsüberkreuzungen charakteristisch waren (Stark 1994). Das Ergebnis der Privatisierung war eine hybride Struktur, die über Personalunionen zwischen privatem Geschäft und Funktionen in staatlichen Unternehmen ${ }^{19}$ oder formale Überkreuzung von Eigentum staatlicher und privater Akteure weit stärker verwoben war als in Polen. Rent-seeking nahm in diesem institutionellen Umfeld die Form der staatlichen Schuldensubventionierung an. Vergleichende Untersuchungen zur Politik der Banken und ihrer Rolle als Akteure eines corporate governance haben gezeigt, daß die staatliche Refinanzierungspolitik in Ungarn -

wurde. (vgl. Balicka 1997). Auch hier stellt sich die Frage, inwieweit das lukrative Importgeschäft durch Lobbies (Universal und Electrim) klientelpolitisch unterstützt, anstatt im Interesse lokaler Kleinproduzenten gedrosselt wird.

18 Mit Wachstumsraten des BSP von über $7 \%$ und der industriellen Produktion von über 15\% liegt Polen auch in der ersten Hälfte des Jahres 1997 an der Spitze der mitteleuropäischen Reformländer (diese Zahlen nach The Economist 14.6.1997, 128).

19 Im Jahr 1993 hatten 40,4\% der Spitzenmanager in ungarischen Staatsunternehmen noch ein privates Unternehmen, während der entsprechende Anteil in Polen bei nur 7,2\% lag (Hanley, Mateju, Vlachova, Krejci 1996, 35). 
und zwar unter der konservativen ebenso wie unter der post-sozialistischen Regierung - weitaus freigiebiger war als in Polen. ${ }^{20}$ Die Studie zeigte darüber hinaus, daß anders als eine einmalige polnische Refinanzierungsmaßnahme, die mit Strukturreformen im Bankwesen und Veränderungen der Anreizsysteme verbunden war, in Ungarn routinemäßige Refinanzierungsmaßnahmen bis Ende 1994 durchgeführt wurden, die nicht mit Reformen des Bankwesens und Maßnahmen zur Effektivierung der Kontrollfunktionen gegenüber den Betrieben verbunden wurden. In der Schwäche der Kreditgeber sahen die Autoren der Studie die Voraussetzungen »spontaner Privatisierung « durch das Management (Baer/Gray 1996, 81ff, 100). Inzwischen wurde in Ungarn seit 1995 die Privatisierung auch im Bankensektor zügig weitergetrieben, so daß die oben beschriebene Politik als Übergangsphänomen ${ }^{21} \mathrm{zu}$ betrachten ist.

Neben der starken Position des Unternehmensmanagements ist eine Besonderheit der ungarischen Entwicklung der extrem hohe Anteil ausländischen Kapitals, der mit $80 \%$ der Privatisierungsmaßnahmen angegeben wird (NZZ 10./11.5.1997, 12). Die ökonomische und soziale Bedeutung dieser Politik können wohl kaum pauschal bewertet werden. Für die Zukunft wird in dem hohen Engagement ausländischen Kapitals und den damit assoziierten Produktivitätsanstiegen eine Wachstumsquelle gesehen. ${ }^{22}$

Das ungarische Selbstbild eines alterfahrenen »Reformvorreiters « akzentuiert die Kontinuität des Wandels und faßt die ökonomische Transformation technokratisch als einen sozialtechnologischen Umstellungsprozeß. Prominentes Instrument des institutionellen Wandels ist nach ungarischem Selbstverständnis die Rechtssetzung. Die Betonung rechtspolitischer Strategien wird auch durch die Formen gesellschaftlichen Interessenausgleiches, etwa im hochformalisierten »Rat für Interessenausgleich«, deutlich (Deppe/ Tatur 1996 und 1997). Dieser Legalismus wird allerdings durch eine Flut von

20 Während es in Polen nur zu einer einzigen Refinanzierung Anfang der neunziger Jahre kam, erfolgten in Ungarn vier Refinanzierungsschübe 1991, 1992, 1993 und im Dezember 1994 (Baer/Gray, 1996, 81ff). Die Refinanzierung der Banken kann da als relativ unproblematisch gelten, wo es um den Abbau der Erblasten der Verschuldungsketten der Betriebe ging, sie wird aber da problematisch, wo neue »schlechte Kredite « vergeben und die Verluste der Banken vom Staat getilgt wurden, wie dies in Ungarn der Fall war.

21 Das Haushaltsdefizit, das sich bis 1994/95 zum zentralen Problem auswuchs und als eine der Ursachen des ausbleibenden Wachstums wahrgenommen wurde, wird in der ungarischen Diskussion nur mit der Sozialpolitik, nicht aber mit diesen Techniken versteckter Subventionierung »spontaner Privatisierung« durch Haushaltsmittel in Verbindung gebracht.

22 Nach Angaben des Wiener Instituts für Internationale Wirtschaftsvergleiche lag schon 1994 der Anteil von Unternehmen mit ausländischer Beteiligung bei über 60\% (des Kapitals) während die entsprechenden Anteile in Polen, der Slowakei und der Tschechischen Republik bei 11,5-12,2\% lagen. Die Zahlen für die Anteile an der Beschäftigung lagen niedriger: bei $37,2 \%$ bzw. 7,5-9,7\%. Der positive Einfluß der Ausländer wird durch den Verweis auf den überproportionalen Anteil an den Investitionen (79\%) und Exporten $(65,5 \%)$ in Ungarn begründet (NZZ 10./11.5.1997, 12). 
Korruptionsskandalen relativiert (Nagy 1996) oder auch durch den Umstand, daß die Gesetzgebung z.B. zur Bankaufsicht als die schlechteste und weichste im mitteleuropäischen Raum klassifiziert wird (Baer/Gray 1996, 79 u. Fn. 14).

Die Ursachen der wirtschaftlichen Wachstumsprobleme in der ersten Hälfte der neunziger Jahre werden von ungarischen Politikern und Ökonomen auf konjunkturpolitischer und nicht auf institutioneller Ebene gesucht. Die hier angedeutete These dagegen ist, daß Ungarn durch einen hohen Grad der Kontinuität des politischen Kapitalismus charakterisiert wird, was auch gewisse »konjunkturpolitische Fehler« erklärt, und dies - und nicht ein »überdimensionierter Sozialstaat« (Kornai 1996) - als eine Erklärung der geringen Wachstumsdynamik heranzuziehen ist. Die Kontinuität institutioneller Muster wiederum erscheint als Resultat der politischen Schwäche einer Gesellschaft, die nach der traumatischen Erfahrung der Niederschlagung der Revolution und des Terrors in den sechziger Jahren erfolgreich in das technokratisch reformierte System inkorporiert und - zumindest in den Unter- und Mittelschichten - atomisiert wurde.

In der Tschechoslowakei hatte es bis 1990 keinen Vorlauf spontaner Privatisierung gegeben und die neue Regierung übernahm 1990 einen zentralistischen Wirtschaftapparat, in dessen Rahmen die Autonomie und Macht der Unternehmensmanager begrenzt war. In der Tschechischen Republik brachte der »Volkskapitalismus « der Voucha-Privatisierung der Regierung zunächst einen beachtlichen Legitimitätsgewinn. Der politische Kapitalismus bediente sich auch hier vor allem einer Vielzahl von Gesetzeslücken, die Raum für Affairen insbesondere im Umfeld der staatlichen Banken und ihrer Kreditpolitik eröffneten. ${ }^{23}$ Diese Machenschaften führten in der Tschechischen Republik zu einer gewissen Delegitimierung der Reformpolitik. ${ }^{24}$ In unserem Zusammenhang ist bedeutsamer, daß die 1996/97 sichtbar werdenden ökonomischen Probleme einen Schatten auf die tschechische Transformationsstrategie warfen. Die sich 1997 dramatisch verschlechternden Wirtschaftsindikatoren ${ }^{25}$ wurden in der tschechischen Dis-

23 Ein Beispiel ist die Komanicky-Affaire. Einer von diesem unbekannten Privatmann geschaffenen Briefkastengesellschaft ohne jegliches Kapital wurden Kredite bewilligt, die zum Kauf von privatisierten Unternehmen genutzt wurden, welche dann wiederum als Sicherung für weitere Kredite und Aufkäufe genutzt wurden. Die Vorwürfe der kritischen Öffentlichkeit richteten sich in der Affaire Komanicky zum einen gegen die Vergabe »fauler« Kredite, und eine Konzentration von Kapital, die einzig und allein durch Kontakte im Banksektor begründet war; und zum anderen gegen die passive Nutzung des erworbenen Kapitals (Vermietung) sowie den demonstrativen Konsum der neuen Reichen.

24 Umfragen zeigten, daß »Unternehmer« mit »Betrüger« assoziert wird, und daß das zunächst umfassende Vertrauen in die ökonomische Transformation zusammenschmolz (Manochin, Tucek u. a. 1996).

25 Hierzu gehörte nicht nur ein (wie überall) dramatisch wachsendes Außenhandelsdefizit, sondern auch ein sich verlangsamendes Wirtschaftswachstums und ein überraschender Rückgang der industriellen Produktion. 
kussion nicht nur mit einer übermäßigen Expansion der Löhne und der Konsumnachfrage, sondern auch einem Formalismus der Eigentumsumgestaltung in Verbindung gebracht. Die Voucha-Privatisierung war weniger als die Umgestaltungen in Ungarn und in Polen mit mikroökonomischen Restrukturierungen verbunden und hatte zu einer Konzentration wirtschaftlicher Macht bei den neu geschaffenen und wenig transparenten Investmentfonds geführt. Diese Fonds, die gleichzeitig als Investoren und Kreditgeber fungieren, hatten - wie sich herausstellte - Teile des verwalteten Vermögens einfach rechtswidrig abgezogen und zum Teil ins Ausland transferiert. Diese Transaktionen wurden auch mit den Bankzusammenbrïchen 1996 in Zusammenhang gebracht. Kritisiert wurde in diesem Zusammenhang, daß das Management der Fonds, keiner effizienten corporate governance unterläge, wodurch die Kleinaktionäre das Nachsehen hätten. In Reaktion auf die Krisensymptome und unter dem Druck einer starken parlamentarischen Opposition hat die Regierung Klaus allerdings nicht nur konjunkturpolitische Maßnahmen, sondern auch eine Novellierung der Gesetze über Investmentfonds und -gesellschaften, sowie des Bankgesetzes und eine stärkere Trennung von Geschäfts- und Investmentbankfunktionen in Aussicht gestellt (NZZ v.12./13.4.1997 und v. 17.4. 1997).

Die kurze Skizze der Privatisierungspolitiken sollte zweierleich deutlich machen: (1) Auch innerhalb des Spektrums der mitteleuropäischen Reformvorreiter lassen sich unterschiedliche Entwicklungen, insbesondere unterschiedliche Agenden und Trägergruppen des politischen Kapitalismus beobachten. (2) Ungeachtet dessen, daß sich in allen drei Ländern unterschiedlich camouflierte Formen des rent-seeking ausmachen ließen, ist dennoch zu konstatieren, daß wir es nicht nur mit einem Transfer von Macht, sondern auch mit einer Transformation der Form von Macht und einer schrittweisen Rationalisierung des politischen Kapitalismus zu tun haben. Das machen die Entwicklungen in Süd- und Osteuropa deutlich, die in eine andere Richtung weisen.

Der institutionelle Wandel in Südosteuropa zeigt nicht nur andere zeitliche Sequenzierungen, sondern auch andere Techniken und Konsequenzen des politischen Kapitalismus. Es gab in Rumänien, Bulgarien oder Albanien keinen Vorlauf der Herausbildung alternativer gesellschaftlicher Akteure und Orientierungen - weder in der Dimension einer relevanten demokratischen Opposition noch in der einer »spontanen Privatisierung « und technokratischen Modernisierung der Nomenklatur. Der Systembruch traf hier atomisierte Gesellschaften und in extremer Weise machiavellistisch denkende Machteliten. Der politische Kapitalismus hat in beiden Ländern stärker extraktive, stärker illegale und kriminelle Züge und war ökonomisch destruktiver als in den mitteleuropäischen Reformländern. Allerdings ist auch in der ökonomischen Destruktion eine gewisse Logik unverkennbar, soweit 
sie der machtpolitischen Umverteilung von Ressourcen diente. Genau das scheint in Rumänien und vor allem in Bulgarien der Fall zu sein, wo die post-kommunistischen Eliten mit einer »gradualistischen« Politik zwar die Chancen einer finanziellen Stabilisierung verspielten und die Gesellschaft in eine Katastrophe steuerten, zugleich aber ansehnliche Kapitalien konzentrieren und handlungsfähige kriminelle Wirtschaftsorganisationen schaffen konnten. ${ }^{26}$ Die »neo-liberale « Schocktherapie, die hier erst Mitte der neunziger Jahre eingeleitet wird, trifft damit auf eine gegenüber 1990 modifizierte, extrem polarisierte Sozialstruktur, d.h. auf starke und durchsetzungsfähige ökonomische Machteliten und eine in äußerster Weise pauperisierte Bevölkerung.

Ein extremes Beispiel ist Albanien, wo sich eine hoch kriminalisierte postsozialistische Umverteilungsökonomie mit offenbarer Unterstützung von ausländischen Mafiaorganisationen, die die albanischen Finanzeinrichtungen für ihre Geldwäsche nutzten, in weit größerem Umfang als andernorts der Technik von Sparspiralen bedienen konnte, um der Bevölkerung ihre letzten Reserven zu entlocken. Als das Unternehmen und der Umfang des Betrugs aufflogen, gelang es den Organisatoren der Spiralen, den Volkszorn gegen eine ohnmächtige Regierung zu lenken, ${ }^{28}$ den staatlichen Gewaltapparat aufzulösen und die latente Anarchie manifest werden zu lassen. Ein anderes extremes Beispiel ist Serbien, wo es die auf den staatlichen Gewaltapparat und die staatlichen industriellen Großbetriebe gestützte Regierung war, die über mehrere Schübe einer gesteuerten Hyperinflation die Ersparnisse einer - zunächst relativ wohlhabenden - Bevölkerung liquidieren konnte. Hinter dem oberflächlichen Gegensatz der anarchischen Implosion des Staates in Kriminalität und Bandenwesen in Albanien und der ungewöhnlichen Kontinuität des Staatsapparates in Serbien, eines Staates allerdings, der im Verlaufe des Krieges selber einer offenen Kriminalisierung

26 In Bulgarien wurden die großen, nach wie vor staatlichen Industriebetriebe offen und versteckt subventioniert, was aber weniger den Beschäftigten in den Dinosauriern des Sozialismus oder gar einer wirtschaftsstrukturellen Anpassung diente, als einem Milieu von korrupten Funktionären und kriminellen Privatunternehmen, das die Unternehmen aushöhlte und staatliche Ressourcen über die Staatsunternehmen in private Hände transferierte. Eine Politik »fauler« Kredite führte 1996 zum Zusammenbruch des Bankensystems, der Währung und der öffentlichen Haushalte und der Stillegung großer Teile der Wirtschaft (NZZ 8.4.1997).

27 Auf den ersten Blick erstaunlich scheint, daß sich die Situation in Bulgarien offenbar dramatisch von der im »Armenhaus« Rumänien unterscheidet, und der Umfang der Zerstörung weit höher ist. Hier stellt sich die Frage, ob der rumänische Nationalismus, der sich so deutlich von der Russophilie der Bulgaren unterscheidet, als Erklärungsfaktor dienen könnte. Bulgarien blieb auch in den neunziger Jahren handelspolitisch und institutionell außerordnentlich stark mit der russischen Ökonomie verflochten.

28 So geschehen in Saranda in Südalbanien, wo einer der Erfinder und Nutznießer der Sparspiralen zum Anführer der Revolte avancierte. 
unterlag, ${ }^{29}$ steckten die gemeinsamen Merkmale extremer Gewaltförmigkeit der Machtausübung und einer ökonomisch zerstörerischen Extraktion aller Ressourcen aus einer atomisierten und ohnmächtigen Bevölkerung. Von serbischen Wirtschaftswissenschaftlern ist in diesem Zusammenhang von einer »Ökonomie der Zerstörung « gesprochen worden. ${ }^{30}$

Auch die Entwicklung Rußlands läßt sich nicht mit Phasen und Richtung des mitteleuropäischen politischen Kapitalismus gleichsetzen. Wie in Südosteuropa erfolgte die Privatisierung in Rußland in einem weitgehend anarchischen, rechtsfreien Raum. Wie dort wurde sie von einer lang anhaltenden Hyperinflation flankiert, die die Ressourcenumverteilung unterstützte. Im Vergleich mit den mitteleuropäischen Reformländern zeichnet sich der russische politische Kapitalismus durch die hohe Gewaltförmigkeit ökonomischer Beziehungen aus und ähnelt in diesem Punkt der Situation in Albanien oder Serbien. Zugleich kam es aber in Rußland weder zur Auflösung noch zur Aufrechterhaltung des staatlichen Gewaltapparates, sondern zu seiner Fragmentierung. Die unübersehbare Besonderheit des russischen politischen Kapitalismus sind parastaatliche Wirtschaftskonglomerate, mit Verankerung im Energiesektor und Finanzwesen (aber auch in der Rüstungsindustrie), die - als hybride Wirtschaftsakteure - zusammen wohl über mehr als die Hälfte des russischen Wirtschaftspotentials verfügen (Slay 1997, 35). Diese oligopolistischen und monopolistischen Wirtschaftsakteure treten tendenziell an Stelle des Staates, ${ }^{31}$ indem sie einen segmentierten Gewaltapparat und die zentralen politischen Institutionen als Machtbastionen nutzen, gegeneinander ausspielen und die staatliche Umverteilungsma-

29 Das betrifft sowohl die ökonomischen Aktivitäten von mit dem Staatsapparat verschwägerten Schmugglerbanden und Waffenhändlern, als auch die Transformation des militärischen Apparates, d.h. die Aufwertung der Freischärler-Banden gegenüber der regulären Armee.

30 Den Hinweis verdanke ich Bodo Weber.

31 Auch wenn dieser noch weite Teile des Produktionsapparates besitzt. Für die Fusion von neuen privaten Akteuren und Staat und den Transfers von Ressourcen und Macht bieten die Banken den Schlüssel. Für das besondere Verhältnis von Banken und Staat ist die Institution der »Bankagenten « ein beredtes Beispiel. 1993 erhielt die Most-Bank das Recht, Auszahlungen des Moskauer Staatsbudgets zu verwalten. Auch die Föderationsregierung ging bald dazu über, Geschäftsbanken die Verwaltung von Haushaltsmitteln zu übertragen. Die Bankagenten strichen nicht nur Provisionen ein, sie konnten die anvertrauten Gelder kurzfristig profitabel anlegen, etwa in risikolosen hochverzinslichen Staatsobligationen. Mit solchen Techniken wurden Finanzmittel umverteilt, die es den Banken ermöglichten, industrielle Unternehmen aufzukaufen. Ende 1995 wurde die Verpfändung von lukrativen Staatsunternehmen gegen Kredite der Banken an den Staat zu einem Hebel der Privatisierung. Angesichts dessen, daß der Staat seine Schulden nicht begleichen konnte, gingen die Unternehmen in Bankbesitz über. Auf diese Weise wurden die Erdölgesellschaften Jukos, Sindaco und Sibneft jeweils in den Besitz der Menatepbank (78\%), der Oneximbank $(51 \%)$ und der Stolitschnibank zusammen mit dem Unternehmer Beresowski (51\%) überführt. Beresowski ebenso wie der Präsident der Oneximbank, Potanin, waren über längere Zeiträume in verantwortlichen Positionen der Regierung (NZZ 25.6.1997). 
schine in ihrem gemeinsamen Interesse manipulieren. ${ }^{32}$ Die großen Konglomerate sind zugleich der Prototyp eines neuen Strukturierungsmuster. Der Soziologe Shlapentokh hat auf die Herausbildung von »Dächern« verwiesen, unter denen »Quasi-Märkte«, ausgestattet mit Finanz- und Handelsagenturen sowie privaten Schutzverbänden, vertikal organisiert werden (Shlapentokh 1996). Shlapentokh hat die neue Formation als »frühfeudalistisch« charakterisiert. Die russische Besonderheit, die diese hierarchisch organisierten Konglomerate meiner Meinung nach von ähnlichen Organisationen in Ostasien unterscheidet, ist die Gewaltförmigkeit der Integration und der Abgrenzung nach außen, die dem Fehlen moralischer Ressourcen der Integration entspricht.

Die ökonomische Funktion dieser segmentierten Machtstruktur ist weder mit »produktiver Zerstörung « noch mit einer reinen »Ökonomie der Zerstörung « gleichzusetzen. Vielmehr haben wir es hier offenbar mit einer Infrastruktur zu tun, die sich auf extraktive Industrien oder basic factors stützt. Schon Anfang der neunziger Jahre haben Krotov und Burawoy auf der Basis von Untersuchungen in der russischen Holzindustrie von einer »neomerkantilistischen « Struktur gesprochen, die nur extraktive Funktionen habe (Burawoy/Krotov 1992 u. 1993). ${ }^{33}$ Man kann Rußlands politischen Kapitalismus mit Burawoy und Krotov (1993) als Hebel der gesellschaftlichen Peripherisierung sehen. Allerdings sind weder die russischen Politiker noch die Energiekonzerne als Marionetten westlicher Interessen mißzudeuten. Die »Kolonialisierung « ist souverän. Ihr Ergebnis ist nicht nur die Peripherisierung der russischen Gesellschaft, sondern auch die Konstitution von hochkonzentrierter Wirtschaftsmacht. Die um die Finanz- und Energiekonzerne sich formierenden Konglomerate sind nicht nur lokale parastaatliche Organisationen, sondern offensive Akteure im globalen »Spiel «. ${ }^{34}$

Die divergierenden Formen des politischen Kapitalismus haben erkennbare sozialstrukturelle Konsequenzen. Vergleichende Untersuchungen aus dem Jahr 1993 (bzw. für Polen 1994) zeigen deutlich divergierende Entwicklungen in Mitteleuropa einerseits und Rußland andererseits (Domanski 1996).

32 Besonders eklatantes Beispiel sind Preispolitik und Steuerbefreiung der Energiekonzerne (Aslund 1996).

33 Ihrer antiliberalen Rhethorik und dem impliziten politischen Vorschlag einer Stützung der Zentristen und des Produktionssektors, d.h. der Schwer- und Rüstungsindustrie (Burawoy/ Krotov 1993), kann ich allerdings nicht folgen. Ursache der Politik der Extraktion sind nicht die neoliberalen Politiken der Reformer, sondern die opportunistischen Orientierungen der Eliten und vor allem die Ohnmacht der Gesellschaft. Die Option für den militärisch-industriellen Konplex müßte keineswegs zwingend eine für die Gesellschaft ökonomisch rationalere Restrukturierung des Produktionsapparates bedeuten. Im Gegenteil, eine in ähnlicher Weise opportunistische Strategie der entsprechenden Segmente der Machteliten könnte - wie das Beispiel Serbien ahnen läßt - weltpolitisch sehr viel gefährlichere und für die russische Bevölkerung kaum günstigere Konsequenzen haben.

34 Gasprom verfügt über 25\% der gesamten Gasvorkommen der Erde. 
Bulgarien, das 1993 als südosteuropäisches Land in die Erhebung einbezogen wurde, zeigt dabei eine mittlere Position zwischen den beiden Typen.

Die Durchschnittsfamilieneinkommen ebenso wie die Durchschnittslöhne und -gehälter lagen in Rußland erheblich unter dem mitteleuropäischen $\mathrm{Ni}$ veau, ${ }^{35}$ zugleich - und das ist das eigentlich Interessante - zeigten sich im Vergleich zu Mitteleuropa weit tiefere Ungleichheiten bei der Verteilung. Während die Spannen zwischen den 10\% höchsten und den 10\% niedrigsten der erfaßten Einkommen in Mitteleuropa zwischen 65:1 in Polen, 42:1 in Tschechien und 32:1 in Ungarn lagen, betrug sie in Rußland 172:1 (Domanski 1996, 76f). Dieser Untersuchung zufolge erschien Ungarn als das reichste und egalitärste Land Mitteleuropas, und Bulgarien nahm eine Zwischenstellung zwischen Rußland und den mitteleuropäischen Ländern ein. Inzwischen dürften die Rückschnitte bei den Reallöhnen 1995 in Ungarn und der Zusammenbruch der bulgarischen Wirtschaft das Bild verändert und Bulgarien an Rußland und Ungarn an Polen angenähert haben.

Gleichzeitig zeigte die schmale russische Oberschicht ein ganz anderes Profil als die breiteren Mittelschichten in Mitteleuropa. Nach westlichen Ansätzen zur Stratifikationsforschung unterscheidet die Studie zwischen »alten« (Unternehmer und Selbständige) und »neuen« (Intelligenz und leitende Angestellte) Mittelschichten. Hinsichtlich der inneren Struktur der post-sozialistischen Mittelschicht zeigt sich danach folgendes Bild: Für Rußland ist eine extreme Spanne zwischen den Einkommen von Intelligenz und Leitungskadern auf der einen und den Verdiensten der neuen Unternehmer und Selbständigen auf der anderen charakteristisch. Die Familieneinkommen der letzten Gruppe übertrafen die der qualifizierten und leitenden Angestellte um das dreifache und mehr. In Ungarn und Polen dagegen lagen die erhobenen Durchschnittseinkommen der Unternehmer und Selbständigen unter denen von Intelligenz und leitenden Angestellten, während die Zahlen für Tschechien und die Slowakei fast gleiche Einkommensverhältnisse für die beiden Kategorien auswiesen. Gleichzeitig wurden die russischen Unternehmer durch einen stärkeren Nomenklaturahintergrund charakterisiert als ihre ungarischen und polnischen Kollegen, die sich in höherem Umfang aus dem sozialistischen Privatsektor entwickelt hatten (Domanski 1996, 74, 103). Ganz offensichtlich handelte es sich um unterschiedliche Kategorien von »Unternehmern « und »qualifizierten und leitenden Angestellten « und einen unterschiedlichen Status der neu herausgebildeten Sektoren. Berücksichtigen wir noch die quantitativen Unterschie-

35 Das durchschnittliche Familieneinkommen pro Kopf lag in Ungarn beim 6,3fachen des russischen Niveaus. Zugleich lag das Verhältnis der ungarischen Familieneinkommen zu dem anderer mitteleuropäischer Länder bei 1,7 für die Slowakei, 1,6 für Polen und 1,4 für Tschechien (Domanski 1996, 75ff). Man kann Zweifel anmelden, ob in diesen Zahlen die unterschiedlichen Lebenshaltungskosten etwa in Polen und der Tschechischen Republik angemessen berücksichtigt ist, die Grundtendenz aber ist eindeutig. 
de, so kann vermutet werden, daß die relativ breite Mittelschicht in Ungarn und Polen sich aus einem breiten, von Kleinbetrieben und Selbstbeschäftigern getragenen Privatsektor und Angestellten im Unternehmenssektor zusammensetzt. In Rußland dagegen, wo es einen sozialistischen Privatsektor nicht gegeben hat, wird die schmale Schicht von Unternehmern und Selbständigen von Transformationsgewinnlern gebildet, während die Beschäftigten im Staatssektor eine absolute und relative soziale Degradierung erfahren. In Rußland haben sich aus einer an die Armutsgrenze abgestiegenen alten sozialistischen Mittelschicht nur die Selbständigen und Unternehmer mit einem Einkommen herausgehoben, die die kaum differenzierten Einkommen von Intelligenz, leitenden Angestellten, Technikern, qualifizierten und nicht qualifizierten Arbeitern um ein Vielfaches übersteigen. ${ }^{36}$ In den mitteleuropäischen Ländern dagegen ist an die Stelle der sozialistischen Mittelschicht ein Stratifikationsmodell getreten, das - anders als in den achtziger Jahren und in Rußland - Bildung und Qualifikation mit Einkommen korrelieren läßt. ${ }^{37}$ Den Aufstieg einer neuen Mittelschicht bestätigen auch spätere Untersuchungen aus der Mitte der neunziger Jahre in Polen. Diese weisen darüber hinaus die hochqualifizierten Angestellten und Selbständigen als eine Gruppe mit starkem bürgerlichen Engagement und liberalen Orientierungen aus (Domanski 1997).

Das folgende Schema versucht die vergleichenden Beispiele und Überlegungen zu systematisieren. Die zuvor eingeführten idealtypischen Kategorien des kriminellen, politischen und demokratischen Kapitalismus können nun als Bezugsmodelle der Richtung des Wandels gedacht werden.

Die Diversifikation der Transformationspfade erklärt sich aus den Akteurskonstellationen Ende der achtziger Jahre und kulturellen Besonderheiten, die zurück in die Geschichte verweisen. In den zwei osteuropäischen der »drei Regionen Europas« (Sücz 1990) lassen sich grob gesprochen drei Pfade unterscheiden: (1) Rußland, das mit der sozialistischen Industrialisierung bäuerliche Muster (Charisma, Kollektiv, moralische Ökonomie) mit den Traditionen des Militärstaates und der Dienstklassengesellschaft verband, und sich zum Kern des »sozialistischen Weltsystems « machen konnte, (2) bäuerliche Gesellschaften, die in den ethnischen Sozialstrukturen Osteuropas bis ins zwanzigste Jahrhundert hinein ausgegrenzt blieben (Belorus, Ukraine, in begrenztem Umfang Bulgarien) und mit der sozialistischen Industrialisierung eine Scheinmodernisierung erfuhren (Jowitt 1992,

36 Das der Unternehmer mit Beschäftigten liegt dabei ungefähr dreimal, das der Selbständigen 4-5mal über dem der anderen Kategorien (Domanski 1996, 82).

37 Die Situation in Tschechien und der Slowakei, wo es einen nennenswerten sozialistischen Privatsektor nicht gegeben hatte, unterscheidet sich von der in Ungarn und Polen durch den höheren Status der neuen Unternehmer und Selbständigen.

38 Der Anteil dieser gutverdienenden »professionals« an der Bevölkerung wird für die großen Städte mit über 14\% angegeben (Domanski 1997). 
Baga/Tatur 1997), und (3) Ständegesellschaften, die Modernisierungs- und Nationenbildungsprozesse vor der sozialistischen Industrialisierung bereits eingeleitet hatten und in denen das oktroyierte System als eine kulturfremde Ordnung von der Gesellschaft abgelehnt wurde (Mitteleuropa, aber auch die Baltischen Staaten).

Neben den Strukturmerkmalen der »langen Dauer« (Braudel) lassen sich auch historisch z.T. »zufällige « Ereignisse als Schlüssel der Diversifikationen von Entwicklungspfaden ausmachen, etwa die Art und Weise der Verarbeitung der Krise des Stalinismus in den fünfziger und sechziger Jahren und ihre Konsequenzen für soziale Figurationen und Akteurskonstellationen in der Umbruchszeit.

Politische Kapitalismen als Modi der Transformation

$\begin{array}{lll}\text { Bezugsmodell } & \text { Bezugsmodell } & \text { Bezugsmodell } \\ \text { krimineller } & \text { politischer } & \text { demokratischer } \\ \text { Kapitalismus } & \text { Kapitalismus } & \text { Kapitalismus }\end{array}$

\begin{tabular}{|c|c|c|c|}
\hline $\begin{array}{l}\text { Transformation } \\
\text { des staatlichen } \\
\text { Gewaltmono- } \\
\text { pols }\end{array}$ & $\begin{array}{l}\text { Auflösung des Gewalt- } \\
\text { monopols: Implosion in } \\
\text { kriminelle Banden oder } \\
\text { Kriminalisierung } \\
\text { des Staates }\end{array}$ & $\begin{array}{l}\text { Segmentierung des } \\
\text { Staates }\end{array}$ & $\begin{array}{l}\text { Transformation } \\
\text { in Rechtsstaat }\end{array}$ \\
\hline $\begin{array}{l}\text { Transformation } \\
\text { der machtpoliti- } \\
\text { schen Repro- } \\
\text { duktionslogik }\end{array}$ & $\begin{array}{l}\text { Kontinuität der } \\
\text { Rationalität der Macht, } \\
\text { Ökonomie der Zerstö- } \\
\text { rung }\end{array}$ & $\begin{array}{l}\text { Transformation in } \\
\text { extraktive Ökonomie }\end{array}$ & $\begin{array}{l}\text { Transformation } \\
\text { in ökonomische } \\
\text { Rationalität, } \\
\text { produktive Zer- } \\
\text { störung, Produkti- } \\
\text { vitätswachstum }\end{array}$ \\
\hline $\begin{array}{l}\text { gesellschaftlicher } \\
\text { Kontext }\end{array}$ & Atomisierung & Segmentierung & Integration \\
\hline $\begin{array}{l}\text { Transformation } \\
\text { der sozialisti- } \\
\text { schen Mittel- } \\
\text { schicht }\end{array}$ & $\begin{array}{l}\text { Auflösung der sozialis- } \\
\text { tischen Mittelschicht } \\
\text { soweit vorhanden), } \\
\text { kriminelle Machtelite } \\
\text { vs. pauperisierte Masse } \\
(90 \%)\end{array}$ & $\begin{array}{l}\text { weitgehende Auflösung } \\
\text { der sozialistischen } \\
\text { Mittelschicht, } \\
\text { Machtelite u. kleine } \\
\text { reiche Unternehmer- } \\
\text { schicht, pauperisierte } \\
\text { Masse }(60 \%)\end{array}$ & $\begin{array}{l}\text { relativ breite alte } \\
\text { und neue Mittel- } \\
\text { schicht, } \\
\text { Pauperisierung } \\
\text { großer Teile der } \\
\text { Bevölkerung } \\
(30 \%)\end{array}$ \\
\hline
\end{tabular}




\section{Schluß}

Das Ausgangsargument dieses Beitrages war die These, daß die sozialistische Ordnung nicht als ein ökonomisches System, sondern als eine Formation zu begreifen sei, in die eine widersprüchliche und selbstzerstörerische Logik der Machterweiterung eingegeben war. Das Projekt der Transformation als Übergang zu Markt und Demokratie sei deshalb als Rekonstitution des Staates, als Institutionalisierung starker Eigentumsrechte und institutionelle Differenzierung der Eigenrationalitäten von Politik und Ökonomie zu modellieren, wobei das Konzept des politischen Kapitalismus auf eine hybride Form des Übergangs verweist.

Die »institutionalistischen« Ansätze der Transformationsforschung und daraus resultierende »gradualistische « Politikstrategien wurden deswegen kritisiert, weil sie einen von der Gesellschaft institutionell abgegrenzten regulativen Rechtsstaat voraussetzen, der empirisch angesichts der Verschränkung von Politik und Ökonomie nicht gegeben ist. Der institutionelle Differenzierungsprozeß ist das Projekt und nicht der Ausgangspunkt der Transformation. Die Frage nach der Herausbildung effizienter demokratischer Institutionen wurde in Kategorien kultureller Kompetenzen und moralischer Ressourcen übersetzt und mit assoziativen und kognitiven Mustern einer zivilen Gemeinschaft in Verbindung gebracht.

In der vergleichenden Skizze haben wir uns auf die Formen des politischen Kapitalismus konzentriert und einen unterschiedlichen Grad der Demoralisierung und kulturellen Fragmentierung eher unterstellt als aufgezeigt. Der Beitrag ist so nur ein erster Schritt zur Entwicklung von Kategorien für eine vergleichende Analyse der Transformationsprozesse in Osteuropa.

Die hier vorgeschlagenen Formulierungen fassen die Rationalisierung ökonomischer und politischer Beziehungen als zivilgesellschaftliche Emanzipation. Die Kategorien, die dabei zur Spezifizierung des Konzeptes des politischen Kapitalismus vorgestellt wurden, lassen sich für die vergleichende Analyse von Ländern, aber auch Mikro-Regionen im osteuropäischen Raum weiterentwickeln. Die methodologische Schlußfolgerung der vorgetragenen Überlegungen ist, daß dabei kulturellen Normen und der moralischen Konstruktion sozialer Beziehungen besondere Aufmerksamkeit zu widmen ist.

Die hier entwickelten Kategorien scheinen aber auch auf politische und ökonomische Ordnungen außerhalb des ehemaligen Ostblocks anwendbar zu sein. In solch einem Verlust der Exklusivität der Begrifflichkeit der Osteuropaforschung drückt sich das Ende der strukturellen »Einmaligkeit« und damit auch der Umfang eines bereits vollzogenen Systemwandels aus. Wenn es aber so sein sollte, daß der regulative Rechtsstaat auch andernorts nicht umstandslos vorausgesetzt werden kann, was in Lateinamerika, der Türkei, Südkorea oder Italien offensichtlich und vielleicht auch bei uns 
möglich ist, besteht dann nicht die generelle Notwendigkeit »to bring society back in $\ll$ ?

\section{Literatur}

Aslund, A. (1995): How Russia Became a Market Economy, Washington DC 1995.

- (1996): Reform vs. »Rent-Seeking « in Russia`s Economic Transformation, in: Transition 2/2 (26.1.1996), 12-16.

Baer, H.L.; Gray, C.W. (1996): Dept as a Control Device in Transitional Economies: The Experiences of Hungary and Poland, in: R.Frydman, C.W.Gray, A.Rapaczynski: Corporate Governance in Central Europe and Russia, Budapest u.a., Bd.I, 68-110.

Baga, E.; Tatur, M. (1997): Rumäniens Sonderweg in Mitteleuropa. Ein Beitrag zu den zivilgesellschaftlichen Ressourcen der Transformationen in Osteuropa, in: Comparativ.

Banfield, E. (1958): The Moral Basis of a Backward Society, Glencoe.

Balcerowicz, L. (1995): Socialism, Capitalism, Transformation, Budapest.

Balicka, M. (1997): Zmurszala deska ratunku, in: Polityka 12.4.1997, 76-78.

Berger, P.L. (1986): The Capitalist Revolution. Fifty Propositions about Property, Equality and Liberty, New York.

Bönker, F. (1995): The Dog that did not Bark? Politische Restriktionen und ökonomische Reformen in den Visegradländern, in: Leviathan Sonderband 15/1995, 180-206.

Burawoy, M.; Krotov, P. (1992): Der Übergang vom Sozialismus zum Kapitalismus in der früheren Sowjetunion: Arbeiterkontrolle und Bargaining in der Ökonomie, in: PROKLA 89, S.508-551.

- (1993): The Economic Basis of Russia`s Political Crisis, in: New Left Review 198, 49-69.

Crawford, B. (1995): Post-Communist Political Economy: A Framework for the Analysis of Reform, in: dies.: Markets, States, and Democracy, San Fransisco, 3-42.

Deppe, R.; Tatur, M. (1995): Transformationssequenzen und Gewerkschaftskonstellationen in Polen und Ungarn, in: Institut für Sozialforschung (Hg.), Mitteilungen 1995/6, 5-34.

- (1996) (Hg.): Ökonomische Transformation und gewerkschaftliche Politik, Umbruchsprozesse in Polen und Ungarn auf Branchenebene, Münster.

Domanski, H. (1996): Na progu konwergencji. Stratyfikacja spoleczna $w$ krajach Europy Srodkowo-Wschodniej, Warszawa.

— (1997): Przyszlosc nalezy do klasy sredniej: ale do »nowej«, nie do »starej«, in: Spleczenstwo otwarte, 1997/3, 7-12.

Frydman, R.; Rapaczynski, A.J.; Earle J.E. u.a. (1993): The Privatization Process in Central Europe, Budapest.

Fukuyama, F. (1995): Konfuzius und Marktwirtschaft. Der Konflikt der Kulturen, München.

Hankiss, E. (1991): The »Second Society«. Is there an Alternative Social Model Emerging in Contemporary Hungary?, in: A. Arato, F. Feher (Hrg.): Crisis and Reform in Eastern Europe, New Brunswick London.

Hanley, E.; Mateju, P.; Vlachova, K.; Kreci, J. (1996): The Making of Post-Communist Elites in Eastern Europe, Working Papers Institute of Sociology, Prag.

Höhmann, H.-H. (1995): Marktwirtschaft ohne Alternative? Aspekte und Bewertungsmaßstäbe der osteuropäischen Wirtschaftstransformation, in: BIOST (Hrg.): Zwischen Krise und Konsolidierung. Gefährdeter Systemwandel im Osten, München, 189-200.

Makarczyk, W. (1978): Struktura spoleczna a warunki zyciowe, Sprawozdanie z badan, IFiS.

Manochin, P.; Tucek, M. u.a. (1996): Ceska spolecnost v transformaci. K promenam socialni struktury, Praha.

Janos, A. C. (1991): Social Science, Communism, and the Dynamics of Political Change, in: World Politics 44, 81-112.

Kornai, J. (1995): Das sozialistische System, Köln.

Kornai, J. (1996): Unterwegs, Essays zur wirtschaftlichen Umgestaltung in Ungern, Marburg. 
Messner, D.; Meyer-Stamer, J. (1993): Die nationale Basis internationaler Wettbewerbsfähigkeit, in: Nord-Süd aktuell, 1.Quartal, 98-111.

Nagy, L. (1996): La Transition Hongoise de 1990 a 1996, Chene-Bourg.

Narojek, W. (1982): Struktura spoleczna $w$ doswiadczeniu jednostki, Warszawa.

North, D.C. (1990): Institutions, Institutional Change and Economic Performance, Cambridge.

North, D.C.; Thomas, R. P. (1973): The Rise of the Western World: A New Economic History, Cambridge.

Offe, C. (1994): Dilemma der Gleichzeitigkeit, in: ders.: Der Tunnel am Ende des Lichts, Frankfurt/M.

Poznanski, K.Z. (ed.) (1995): The Evolutionary Transitition to Capitalism, San Fransisco.

Putnam, R.D. (1993): Making Democracy Work, Civic Traditions in Modern Italy, Princeton.

Rosaty, D.K. (1993): Poland glass half empty, in: R. Portes (ed.): Economic Transformation in Central Europe: A Progress Report, E.C. Luxenburg.

Shlapentokh, V. (1996): Early Feudalism - The Best Parallel for Contempory Russia, in: Europe Asia Studies, Vol.48, No.3, 393-411.

Slay, B. (1997): The Search for Solutions in Enerprise restructuring, in: Transition 10.1.1997, 32-35.

Staniszkis, J. (1984): Poland's Self-limiting Revolution, New York.

- (1991): The Dynamics of Breakthrough in Eastern Europe. The Polish Experience, Berkeley u.a.

- (1991a): Dilemmata der Demokratie in Osteuropa, in: R.Deppe u.a. (Hrg.): Demokratischer Umbruch in Osteuropa, Frankfurt.

- (1992): The Ontology of Socialism, Oxford (polnisch 1989).

- (1994/95): The Politics of Post-communist institutionalization in historical perspective. Advanced Study Center, International Institute University of Michigan, Working Papers Series No.1.

- (1995): In Search of a Paradigm of Transformation, in: E. Wnuk-Lipinski (ed.), After Communism. A Multidisciplinary Approach to Radical Social Change, Warsaw 19-56.

- (1996): Polityka postkomunistycznej instytucjonalizacji w perspektywie histrorycznej, in: Studia Polityczne 1996/4 und 1996/5.

Stark, D. (1991): Privatization in Hungary: From Plan to Market or from Plan to Clan, in: East European Politics and Societies 1991/1.

- (1994): Nicht nach Design: Rekombiniertes Eigentum im osteuropäischen Kapitalismus, in: PROKLA 94, 127-142.

Szelenyi, I.; Treiman, D.; Wnuk-Lipinski, E. (eds.) (1995): Elity w Polsce, w Rosji $i$ na Wegrzech. Wymiana czy reprodukcja? Warszawa.

Szelenyj, I.; Szelenyj, S. (1995): Wstep - Zalozenia teoretyczne i metodologia, in: Szeleny, I. u. a. (1995), 7-34.

Sztompka, P. (1994): Teorie zmian spolecznych a doswiadczenia polskiej transformacji, in: studia socjologiczne 1994/1.

- (1993): Civilizational Incompetence: The Trap of Post-Communist Societies, in: Zeitschrift für Soziologie Jg.22 Heft 2, 85-95.

Szücz, J. (1990): Drei historische Regionen Europas, Frankfurt.

Tarkowska, E.; Tarkowski, J. (1990): »Amoralny familizm« czyli o dezintegracji spolecznej w Polce lat osiemdziesiatych, in: E.Wnuk-Lipinski (Hrsg.): Grupy $i$ wiezi spoleczne w systemie monocentrycznym, Warszawa.

Tatur, M. (1989): Solidarnosc als Modernisierungsbewegung. Sozialstruktur und Konflikt in Polen, Frankfurt.

— (1991a): Zur Dialektik der »civil society« in Polen, in: R.Deppe u.a. (Hrg.): Demokratischer Umbruch in Osteuropa, Frankfurt.

- (1995): Interessen und Norm. Politischer Kapitalismus und die Transformation des Staates in Polen und Ungarn, in: Leviathan Sonderheft 15/1995, 93-116. 
Wnuk-Lipinski, E. (1985) (Hrg): Nierownosci spoleczne - poczucie wiezi - wiara w siebie, Forschungsbericht, IFiS Warszawa, in veränderter Form neu aufgelegt als: derselbe (Hrg.) Grupy i wiezi spoleczne w systemie monocentrycznym, Warszawa 1990.

World Bank (1996): From Plan to Market, World Development Report, Oxford u.a.

\title{
The Berlin Institute of Critical Theory (InkriT)
}

\author{
Invitation and call for papers
}

Discuss with Paul Anthony Bove, Joseph Buttigieg, Iring Fetscher, Marta Gimenez, Frigga Haug, Gayatri Spivak, Bastiaan Wielenga, Frieder O. Wolf, Erich Wulff, and many others at the III. International InkriT-Conference in cooperation with the USAmerican journal boundary 2 (May 13-16, 1999, Jagdschloss Glienicke near Berlin):

\section{Rethinking Progress}

»The concept of progress should be grounded in the idea of catastrophe.« This sentence of Walter Benjamin has been understood as an argument for dropping the idea of progress altogether, instead of grounding it in a new way. The absence of criteria for what constitutes 'sustainable progress' has contributed to the disorientation and weakness of the left. Recuperating critical-progressive competence is a key question under conditions of global neoliberal hegemony. »That things 'just keep on going' is the catastrophe.

Under the general heading Rethinking Progress a wide range of subjects and aspects may be delt with. Among others there will be sections on Varieties of Capitalism, New Forms of Subject(ivitie)s. Half a day will be dedicated to workshops on entries for vol. 4 of the Historisch-kritisches Wörterbuch des Marxismus - among others: progress.

Conference languages are English \& German. No more than 80 participants can be admitted. The conference fee will be 50 US-Dollars. The Glienicke castle offers accomodation plus 3 meals and coffee \& cake during the breaks for 80DM (about 48 US-\$) a day. As to travel \& accomodation expenses, we cannot promise any funding, but we shall try to help at least graduate students and those who are coming from East Europe or from developing countries.

Please send your application and, if you want to present a paper, a short summary of your presentation (preferably in English) to: InkriT, Krottnaurerstr. 72, D-14129 Berlin, Fax +49 - 30 - 8037382 -- E-mail: HKWMred@zedat.fu-berlin.de 